\title{
Cell death mechanisms of the anti-cancer drug etoposide on human cardiomyocytes isolated from pluripotent stem cells
}

\author{
Harshal Nemade ${ }^{1}$. Umesh Chaudhari ${ }^{1}$. Aviseka Acharya ${ }^{1}$. Jürgen Hescheler ${ }^{1}$. Jan Georg Hengstler ${ }^{2}$. \\ Symeon Papadopoulos ${ }^{3} \cdot$ Agapios Sachinidis $^{1}$
}

Received: 10 October 2017 / Accepted: 31 January 2018 / Published online: 3 February 2018

(c) The Author(s) 2018. This article is an open access publication

\begin{abstract}
Etoposide (ETP) and anthracyclines are applied for wide anti-cancer treatments. However, the ETP-induced cardiotoxicity remains to be a major safety issue and the underlying cardiotoxic mechanisms are not well understood. This study is aiming to unravel the cardiotoxicity profile of ETP in comparison to anthracyclines using physiologically relevant human pluripotent stem cell-derived cardiomyocytes (hPSC-CMs). Using xCELLigence real-time cell analyser (RTCA), we found that single high dose of ETP induces irreversible increase in hPSC-CMs beating rate and decrease in beating amplitude. We also identified 58 deregulated genes consisting of 33 upregulated and 25 downregulated genes in hPSC-CMs after ETP treatment. Gene ontology (GO) and pathway analysis showed that most upregulated genes are enriched in GO categories like positive regulation of apoptotic process, regulation of cell death, and mitochondria organization, whereas most downregulated genes were enriched in GO categories like cytoskeletal organization, muscle contraction, and $\mathrm{Ca}^{2+}$ ion homeostasis. Moreover, we also found upregulation in 5 miRNAs (has-miR-486-3p, has-miR-34c-5p, has-miR-4423-3p, has-miR-182-5p, and has-miR139-5p) which play role in muscle contraction, arginine and proline metabolism, and hypertrophic cardiomyopathy (HCM). Immunostaining and transmission electron microscopy also confirmed the cytoskeletal and mitochondrial damage in hPSCCMs treated with ETP, as well as noticeable alterations in intracellular calcium handling and mitochondrial membrane potential were also observed. The apoptosis inhibitor, Pifithrin- $\alpha$, found to protect hPSC-CMs from ETP-induced cardiotoxicity, whereas hPSC-CMs treated with ferroptosis inhibitor, Liproxstatin-1, showed significant recovery in hPSC-CMs functional properties like beating rate and amplitude after ETP treatment. We suggest that the damage to mitochondria is a major contributing factor involved in ETP-induced cardiotoxicity and the activation of the p53-mediated ferroptosis pathway by ETP is likely the critical pathway in ETP-induced cardiotoxicity. We also conclude that the genomic biomarkers identified in this study will significantly contribute to develop and predict potential cardiotoxic effects of novel anti-cancer drugs in vitro.
\end{abstract}

Keywords Non-animal testing · Cardiotoxicity · Safety assessment $\cdot$ Human pluripotent stem cells · Cardiomyocytes · Apoptosis $\cdot$ Calcium

Electronic supplementary material The online version of this article (https://doi.org/10.1007/s00204-018-2170-7) contains supplementary material, which is available to authorized users.

Agapios Sachinidis

a.sachinidis@uni-koeln.de

1 Institute of Neurophysiology and Center for Molecular Medicine Cologne (CMMC), University of Cologne (UKK), Robert-Koch-Str. 39, 50931 Cologne, Germany

2 Leibniz Research Centre for Working Environment and Human Factors, Technical University of Dortmund (IfADo), 44139 Dortmund, Germany

3 Center of Physiology and Pathophysiology, Institute of Vegetative Physiology, University of Cologne, Robert-Koch-Str. 39, 50931 Cologne, Germany

\section{Introduction}

Etoposide (ETP) is a podophyllotoxin derivative with a broad spectrum of antitumor activity, reported as one of the most active single agents in small-cell bronchus carcinoma and is very active in testicular carcinoma, choriocarcinoma and neuroblastoma. ETP inhibits cancer cell growth mainly via inhibition of Topoisomerase II (TopII) resulting in single and double-stranded breaks. It binds reversibly to TopoII $\alpha / \beta$ to block religation of the cleaved DNA strand, and as the binding is transient and non-covalent, enzyme activity is rapidly restored upon clearance of the compound and religation of DNA is completed (D'Arpa and Liu 1989; Soubeyrand 
et al. 2010; Willmore et al. 1998). It also reported to inhibit nucleoside transfer of DNA and RNA synthesis, protein synthesis, and microtubular assembly (Schmoll et al. 1981).

In clinic, ETP in combination with cyclophosphamide and vincristine (CEV) showed no cardiotoxicity and no potentiating neurotoxicity compared to Doxorubicin (DOX) in combination with cyclophosphamide and vincristine (CDV) in the treatment of small-cell lung cancer (SCLC) (Comis 1986; Hong et al. 1989). Similarly, ETP in combination with several other drugs like lomustine, methotrexate, and prednisone also proposed to be first-line therapy in patients with non-Hodgkin's lymphoma (NHL) with no major cardiotoxicity (Dorigo et al. 1993). The previous studies have reported that ETP as a cardio-safe drug with minimum or no cardiotoxicity unlike other Topo II inhibitors like DOX, Daunorubicin (DAUNO), and Mitoxantrone (MITO). However, some reports indicate ETP-induced cardiotoxic side effects with hypotension being the most common (Cohen et al. 1977) along with development of myocardial infarction and vasospastic angina (Bernard et al. 2006; Escoto et al. 2010; Rodriguez et al. 1995; Yano and Shimada 1996). ETP has also been reported to have synergistic toxic effects on cardiac electrical activity when combined with Cisplatin (Canobbio et al. 1986; Petrella et al. 1989; Shoji et al. 2006). These inconclusive findings not only demands more efforts towards understanding the cardiotoxicity associated with ETP, but also highlights the limitations of current in vitro cardiotoxicity models.

Even though various in vitro and in vivo assays have been developed to assess the cardio safety of lead compounds, they often fail to correctly predict the actual adverse cardiac effects because of interspecies variations or lack of humanrelevant model systems. Levels of diagnostic biomarkers like cTnT and cTnI in blood can be assessed easily, but they are released only after chronic cardiac tissue damage and proved to be inconclusive. For example, DOX-induced myocardial damage may appear in endomyocardial biopsy specimens, but may not produce any measurable rise in $\mathrm{cTnT}$ or $\mathrm{cTnI}$ protein levels in blood (Yeh et al. 2004). Hence, there is also an urgent need for novel and reliable in vitro as well as in vivo biomarker assay that will enable immediate and extensive assessment of cardiotoxicity.

We undertook this study aiming, to unravel the cardiotoxicity profile of ETP in comparison to that of DOX, and to further appraise the in vitro biomarkers, we recently reported for anthracycline-induced cardiotoxicity. To achieve this, we used a human-relevant model, applying that human-induced pluripotent stem cells (hiPSC) derived cardiomyocytes (hiPSC-CMs or CMs) and set of in vitro biomarkers constituting differentially regulated 84 genes and 14 miRNAs (Chaudhari et al. 2016a, b) as well as metabolites (Chaudhari et al. 2017). More recently, we could predict cardiotoxicity of cosmetic compounds using the anthracycline regulating genomic biomarkers in combination with functional readout assays (Chaudhari et al. 2018). Here, the hiPSC-CMs were exposed to high single doses of 10,15 , and $30 \mu \mathrm{M}$ of ETP for $48 \mathrm{~h}$. Cardiotoxicity in terms of changes in parameters such as cell index (CI), beating rate, and beating amplitude was monitored by xCELLigence Real-Time Cell Analyser (RTCA) cardio system. Identification of differentially regulated genes and miRNA was achieved using custom $\mathrm{RT}^{2}$ profiler PCR arrays and quantitative real-time PCR (qRT-PCR), respectively. Cellular toxicity was assessed with LDH leakage assay, measurements of the cellular ATP and reactive oxygen species (ROS). The effects of ETP on cell morphology and cellular cytoskeleton structure have been assessed by immunostaining and transmission electron microscopy (TEM). The effects of ETP on calcium handling and mitochondrial membrane potential have been investigated using Rhod-2AM and JC-1 fluorescence imaging.

\section{Materials and methods}

\section{Chemicals}

Etoposide (E1383) was obtained from Sigma-Aldrich Chemie GmbH (Germany), Liproxstatin-1 (S7699) was obtained from Selleckchem (Germany), and Pifithrin- $\alpha$ hydrobromide (2653) was obtained from Tocris (United Kingdom). $10 \mathrm{mM}$ stock solution (in DMSO) was made and stored as small volume aliquots in tightly sealed sterile tubes at $-80^{\circ} \mathrm{C}$. Drug dilutions were performed in pre-warmed $\left(37^{\circ} \mathrm{C}\right)$ iCell-MM prior to each drug exposure.

\section{Cardiomyocyte cell culture}

Purified human iCell Cardiomyocytes ${ }^{\circledR}$ (Cellular Dynamics International, Madison, WI, USA), which are derived from hiPSCs, were used for all the experiments. The cardiomyocytes were supplied as a cryopreserved single cell suspension of $\sim 98 \%$ pure population comprising mixture of electrically active atrial-, nodal-, and ventricular-like myocytes. These cells exhibit typical biochemical, electrophysiological, and mechanical characteristics of normal human heart cells with expected responses upon exposure to exogenous agents. For functional studies, cryopreserved hiPSCCMs were thawed in iCell cardiomyocytes plating medium (iCell-PM, Cellular Dynamics International, Madison, WI, USA) and directly plated on a fibronectin-coated (5 $\mu \mathrm{g} /$ $\mathrm{cm}^{2}, 2 \mathrm{~h}$ at $37^{\circ} \mathrm{C}$ ) E-plate Cardio 96 (ACEA Biosciences, San Diego, CA, USA) at approximately a $25 \times 10^{3}$ cells per well density using iCell-PM. For other studies, thawed cells were plated on fibronectin-coated $\left(5 \mu \mathrm{g} / \mathrm{cm}^{2}, 2 \mathrm{~h}\right.$ at $\left.37{ }^{\circ} \mathrm{C}\right)$ 6-well or 96-well plates at a density of $0.4 \times 10^{6}$ or $25 \times 10^{3}$ cells per well, respectively. From day 2 onwards, cells were 
maintained in iCell cardiomyocyte Maintenance Medium (iCell-MM, Cellular Dynamics International, Madison, WI, USA), with a fresh medium change after every 2 days. The cardiomyocytes were cultured in a standard cell culture incubator at $5 \% \mathrm{CO}_{2}, 37^{\circ} \mathrm{C}$.

\section{The xCELLigence RTCA Cardio system}

The xCELLigence RTCA Cardio system (ACEA Biosciences, San Diego, CA, USA) is an impedance-based platform for monitoring the real-time beating function of cardiomyocytes. The E-plate Cardio 96 (ACEA Biosciences, San Diego, CA, USA) xCELLigence plates were equilibrated using iCell-PM ( $50 \mu \mathrm{l}$ per well) and inserted into the $\mathrm{xCEL}$ Ligence station to measure background impedance and to ensure that all wells and connections were working within acceptable limits. After equilibration, the cells were harvested and seeded in required density. Impedance measurements were monitored at regular time intervals. The amount of growth area covered in an E-plate Cardio 96 due to cell adhesion was represented as the Cell Index (CI). A high CI indicates more cell adhesion and vice versa. The raw data and statistical information, such as mean and SD for the parameters like CI, beating rate, amplitude, normalized CI, normalized beating rate, and normalized amplitude, were acquired using RTCA Cardio software version 1.0 (ACEA Biosciences, Inc., San Diego, CA, USA).

\section{$\mathrm{RT}^{2}$ profiler arrays for mRNA expression analysis}

To analyze the mRNA expression, cell samples were homogenized with QIAzol lysis reagent (QIAGEN, Hilden, Germany), and the total RNA was extracted using the miRNeasy Mini Kit (QIAGEN, Hilden, Germany) according to the manufacturer's instructions. Extracted RNA was assessed for purity and quantity using Nanodrop (ND-1000, Thermo Fisher, Germany). 300-500 ng of total RNA was used for cDNA synthesis using RT ${ }^{2}$ First Strand kit (Qiagen, Hilden, Germany) according to the manufacturer's instructions. Quantitative comparison of mRNA levels was performed using custom made $\mathrm{RT}^{2}$ Profiler PCR array (96-well plate) (Qiagen, Arch Toxicol Hilden, Germany) containing 84 target genes, 5 housekeeping genes, 1 genomic DNA control, 3 reverse transcription controls, and 3 positive PCR controls (all 84 target genes are shown in Supplementary Table No. 1). The qRT-PCR was performed using $R^{2}{ }^{2} S \mathrm{BR}^{\circledR}$ Green ROX $^{\mathrm{TM}}$ qPCR master mix (Qiagen, Hilden, Germany) in an Applied Biosystems 7500 FAST Real-Time PCR System in accordance with the manufacturer's recommended thermal cycling conditions. The relative gene expression analysis was performed using the $2^{-\Delta \Delta C t}$ method with the $\mathrm{RT}^{2} \mathrm{PCR}$ array data analysis online tool. Expression data were normalized using the geometric mean of five housekeeping genes, ACTB, B2M, GAPDH, HPRT1, and RPLPO. A cut-off fold change value of 1.9 was set for significantly deregulated genes and later used to generate the gene list used for Venn diagram analysis.

\section{Quantitative real-time PCR for microRNA expression analysis}

The microRNA expression analysis was performed as we previously reported (Chaudhari et al. 2016a). In brief, $500 \mathrm{ng}$ of total RNA was used for cDNA synthesis with qScript $^{\mathrm{TM}}$ microRNA cDNA Synthesis Kit (Quanta Biosciences, Gaithersburg, USA) following the manufacturer's instructions. The cDNA was diluted as per manufactures instructions and used as a template for qPCR. The amplification of miRNA was performed using the PerfeCTa ${ }^{\circledR}$ microRNA assay primer, the PerfeCTa ${ }^{\circledR}$ Universal PCR primer and the PerfeCTa ${ }^{\circledR}$ SYBR $^{\circledR}$ Green SuperMix, Low ROX ${ }^{\mathrm{TM}}$ Kit (Quanta Biosciences, Gaithersburg, USA) as per the manufacturer's instructions. Quantitative PCR was carried out on an Applied Biosystems 7500 FAST Real-Time PCR System. Relative miRNA levels were calculated using the $\Delta \Delta C_{\mathrm{t}}$ method, and RNU6 was used as the miRNA PCR control.

\section{Lactate dehydrogenase leakage assay}

Cell cytotoxicity/membrane damage induced by ETP was assessed by lactate dehydrogenase (LDH) leakage into the culture medium. The iCell-CMs were seeded on a fibronectin-coated $\left(5 \mu \mathrm{g} / \mathrm{cm}^{2}, 2 \mathrm{~h}\right.$ at $\left.37^{\circ} \mathrm{C}\right) 96$-well plate at a cell density of $20 \times 10^{3}$ cells per well in quadruplets. Cells then exposed to 10, 15, and $30 \mu \mathrm{M}$ of ETP for $48 \mathrm{~h}$. Following the exposure, the culture medium was aspirated and centrifuged at $3000 \mathrm{rpm}$ for $5 \mathrm{~min}$ to obtain a cell free supernatant. The activity of LDH released into the medium was estimated using Pierce ${ }^{\mathrm{TM}}$ LDH Cytotoxicity Assay Kit (Thermo Scientific ${ }^{\mathrm{TM}}$ ) according to the manufacturer's instructions. The absorbance from formazan at $490 \mathrm{~nm}$ was recorded using a Softmax Pro M5e 96-well plate reader (Molecular Devices, Sunnyvale, CA, USA). The raw data were analyzed and presented as percent cytotoxicity with respect to that of control with \pm SD.

\section{Measurement of ATP in cell extracts}

ATP is an important marker for cell viability and present in all metabolically active cells. Its concentration declines very rapidly when the cells undergo necrosis or apoptosis. To measure the changes in intracellular ATP after ETP treatment, cell extracts for hPSC-CMs treated with 10, 15, and $30 \mu \mathrm{M}$ for $48 \mathrm{~h}$ were analyzed using ATPlite ${ }^{\mathrm{TM}}$ assay kit (PerkinElmer, Germany) according to the manufacturer's 
instructions. The light intensity produced was measured at $560 \mathrm{~nm}$ using Softmax Pro M5e 96-well plate reader (Molecular Devices, Sunnyvale, CA, USA). Data were analyzed and represented as percent ATP as compared to that of control.

\section{Immunostaining}

To identify whether the treatment of ETP caused any alterations in cytoskeletal assembly of CMs, we performed immunofluorescence staining of cardiac-specific proteins. Cells from ETP-treated and control groups were fixed with icecold $99 \%$ Methanol at $-20{ }^{\circ} \mathrm{C}$ for 10 min followed by permeabilization and fixing of cells with $0.3 \%$ Triton X-100 for $20 \mathrm{~min}$ and with 5\% Bovine Serum Albumin for $1 \mathrm{~h}$ at room temperature, respectively. Cells were then incubated with anti-sarcomeric alpha actinin ( $\alpha$-actinin) and anti-cardiac troponin $\mathrm{T}(\mathrm{cTnT})$ antibodies at $37{ }^{\circ} \mathrm{C}$ for $1 \mathrm{~h}$. All the antibodies were purchased from Abcam (Abcam, Cambridge, UK) and diluted to working concentration of 1:200. After three washes with $\mathrm{PBS}^{+/+}$, primary antibodies were then detected using species-matched respective Alexa Fluor-488/568-conjugated secondary antibodies (Invitrogen, Darmstadt, Germany) with $1 \mathrm{~h}$ incubation at $37^{\circ} \mathrm{C}$. The cells were washed three times with $\mathrm{PBS}^{+/+}$for 5 min and then mounted with Prolong ${ }^{\circledR}$ Gold anti-fade mount with DAPI (Invitrogen, Darmstadt, Germany). The images were acquired with an Axiovert 200 fluorescence microscope and Axiovision 4.3 software (Carl Zeiss).

\section{Measurement of ROS}

Dihydroethidium (DHE) staining was used to assess the production of ROS in hiPSC-CMs after ETP treatment. DHE is a lipophilic, cell-permeable dye that undergoes oxidation in the presence of superoxide to form ethidium bromide, which then forms an irreversibly complex with the double-stranded DNA. The amplified red fluorescence of ethidium then can be visualized under microscope as a punctate nuclear staining indicating ROS production (Benov et al. 1998; Nijmeh et al. 2010). The ETP-treated hiPSC-CMs were incubated with DHE $(5 \mu \mathrm{M})$ for $30 \mathrm{~min}$ at room temperature. Excess DHE was then washed out and cells were mounted onto a slide with Prolong ${ }^{\circledR}$ Gold anti-fade mount with DAPI (Invitrogen, Darmstadt, Germany). The images were acquired with an Axiovert 200 fluorescence microscope and Axiovision 4.3 software (Carl Zeiss).

\section{Transmission electron microscopy}

For TEM study, hiPSC-CMs were seeded onto fibronectincoated ACLAR ${ }^{\circledR}$ embedding film (Ted Pella, Inc. USA) in 96-well plate and exposed to ETP for $48 \mathrm{~h}$. Post-drug exposure cells were washed with DPBS $(+/+)$ and fixed using $2 \%$ glutaraldehyde and $2 \%$ formaldehyde in $0.1 \mathrm{M}$ cacodylate buffer ( $\mathrm{pH} 7.3$ ) for $2 \mathrm{~h}$ at $4{ }^{\circ} \mathrm{C}$, post-fixed in $1 \%$ OsO4 for $30 \mathrm{~min}$ at $4{ }^{\circ} \mathrm{C}$ in dark. After washing, cell samples were dehydrated in graded ethanol and embedded in to Epon (Sigma) (20 g Epoxy, 11 g DDSA, 9 g NMA, 0.8 g DMP-30) for $48-72 \mathrm{~h}$ at $62^{\circ} \mathrm{C}$. Ultrathin $(\sim 70 \mathrm{~nm})$ sections were obtained using Leica EM UC6 Ultramicrotome (Leica, Germany) and double-stained with uranyl acetate followed by lead citrate. Ultrastructural analysis was done using a transmission electron microscope (EM109, Zeiss, Germany) equipped with frame transfer CCD camera.

\section{Calcium imaging}

The hiPSC-CMs were seeded onto fibronectin-coated thin glass bottom $(0.085-0.115 \mathrm{~mm}) 35 \mathrm{~mm}$ dish (In Vitro Scientific) and treated with ETP for $48 \mathrm{~h}$. Then, the cells were washed and incubated with $2 \mu \mathrm{M}$ Rhod-2, AM Ca ${ }^{2+}$ dye (Invitrogen) for $30 \mathrm{~min}$ at $37{ }^{\circ} \mathrm{C}$, and washed. Rhod2-labeled cells were then observed and fluorescence imaging was performed at room temperature, with an inverted confocal laser-scanning microscope (FV1000, Olympus) operating in the line-scan mode, equipped with a $60 \times$ oil immersion objective. Upon recording a line scans, background subtraction was applied for all data sets, by defining the average (auto-) fluorescence intensity of an extracellular area as the background. The data were then subjected to a fitting procedure which adjusted the parameters of a Weibull function implemented in Sigma Plot (Version 8.04, SPSS). A self-made macro in Excel (Microsoft) calculated the $\mathrm{Ca}^{2+}$ transient amplitude $\left(F / F_{0}\right.$, where $F_{0}$ is the averaged background-corrected resting fluorescence intensity), timeto-peak (TTP), the maximum steepness ( $[\Delta F / \Delta T] \max )$, full-width at half-maximum (FWHM) and T90\%. Data were represented as \pm SEM of 25 measurement $(n=25)$ for each group.

\section{Mitochondrial membrane potential $(m \Delta \psi)$ assay}

We used JC-1-staining method (Nuydens et al. 1999) to investigate the loss of mitochondrial membrane potential in response to ETP treatment $(10,15$, and $30 \mu \mathrm{M})$ for $48 \mathrm{~h}$, DOX $(1 \mu \mathrm{M})$ was used as a positive control. In brief, hiPSC-CMs were seeded onto fibronectin-coated thin glass bottom (0.085-0.115 mm) $35 \mathrm{~mm}$ dish (In Vitro Scientific) and treated with ETP for $48 \mathrm{~h}$. Then, the cells were washed and incubated with $10 \mu \mathrm{M} \mathrm{JC}-1$ dye (AAT Bioquest, CA) diluted in fresh medium for $30 \mathrm{~min}$ in $37{ }^{\circ} \mathrm{C}, 5 \% \mathrm{CO}_{2}$ incubator, and washed. JC-1 emissions after excitation at the $488 \mathrm{~nm}$ were captured at $525 \mathrm{~nm}$ (JC-1 monomers; green) and $590 \mathrm{~nm}$ (JC-1 aggregates; orange). The images were 
captured using inverted confocal laser-scanning microscope (FV1000, Olympus).

\section{Statistical analysis}

Experimental results are expressed as mean \pm SEM. Twotailed Student's $t$ test was used to calculate statistical significance and $\mathrm{p}$ values $\leq 0.05$ were considered as statistically significant.

A

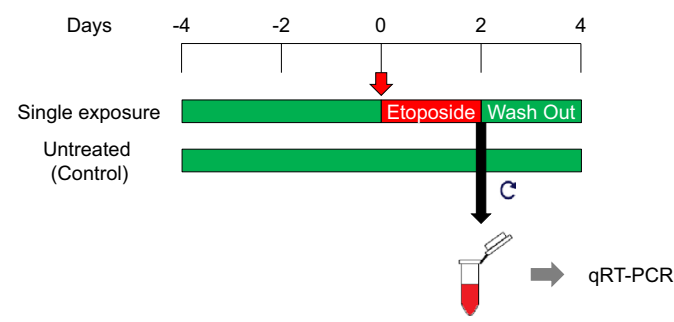

B

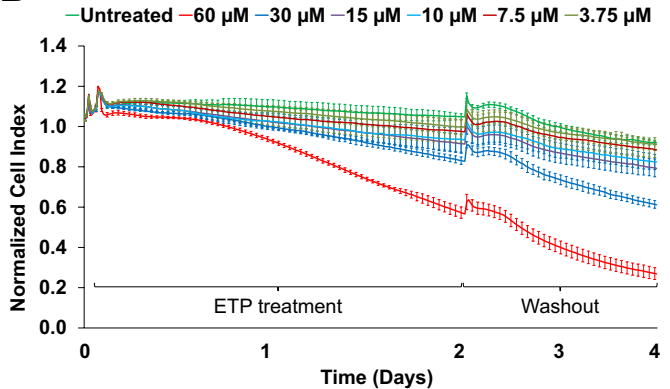

C

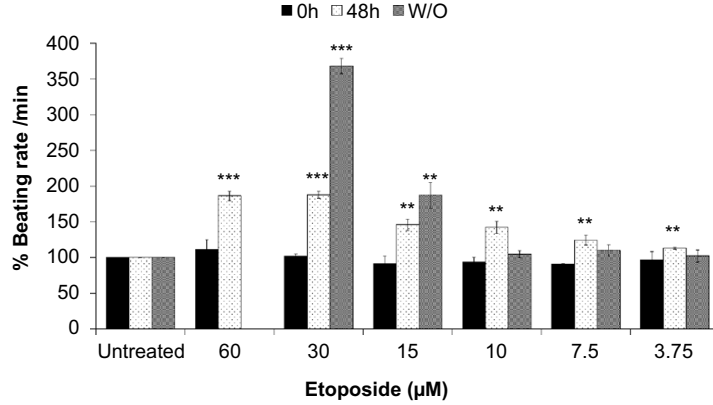

Fig. 1 Single high dose of etoposide induces arrhythmic beating and cytotoxicity in hiPSC-CMs. a Schematic representation and experimental setup of the in vitro cardiotoxicity test model. For functional studies, the synchronously beating hiPSC-CMs in the E-plate Cardio 96 were exposed to ETP (single high-dose exposure) for $48 \mathrm{~h}$. After exposure, the ETP was washed out and the cells were further incubated for $48 \mathrm{~h}$. The effects of ETP on hPSC-CMs functional characteristics were monitored by the xCELLigence RTCA Cardio system. For qRT-PCR studies, RNA from ETP-treated and untreated control cells were harvested at day 2 . b-e Functional studies of ETP-treated hiPSC-CMs. The representative graphs display, b nor-

\section{Results}

\section{Single high dose of etoposide leads to arrhythmic beating and cytotoxicity in hiPSC-CMs}

The hiPSC-CMs were thawed and seeded onto fibronectincoated E-Cardio plate in iCell-PM and maintained in iCellMM. Then, synchronously beating hPSC-CMs treated with ETP according to the timeline, as represented in Fig. 1a. The hPSC-CMs were treated with ETP from day 0 to day 2

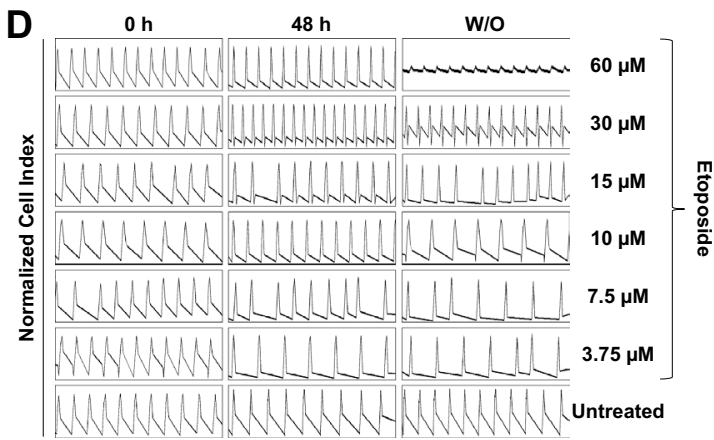

E
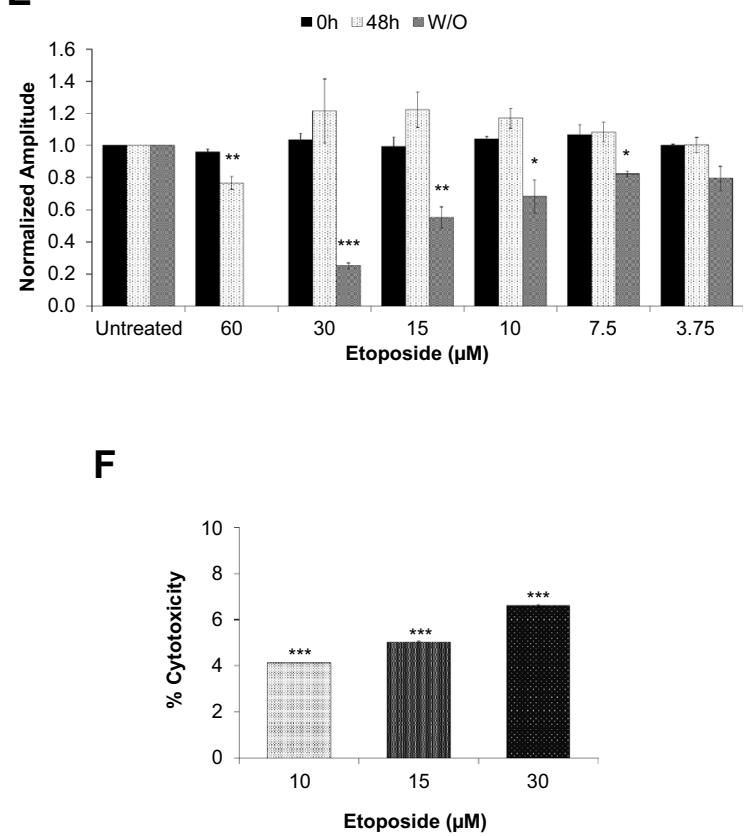

malized CI values showing ETP-induced cytotoxicity $(n=3$, error bars represent \pm SEM), c $\%$ beating rate alterations induced by single dose of ETP in hiPSC-CMs $(n=3$, error bars represent \pm SEM $)$ ( $t$ test, $* p<0.05, * * p<0.01, * * * p<0.001)$, d representative $12 \mathrm{~s}$ beating traces of hiPSC-CMs before, during and after the ETP treatment, e normalized amplitude showing significant drop after ETP treatment $(n=3$, error bars $= \pm \operatorname{SEM})(t$ test, $* p<0.05, * * p<0.01$, $* * * p<0.001)$. f ETP-induced cytotoxicity was assessed by LDH leakage assay. The graph shows \% cytotoxicity induced by ETP compared to untreated control $(n=3$, error bars represent \pm SEM) $(t$ test, $* p \leq 0.05, * * p \leq 0.01, * * * p \leq 0.001)$ 
followed by $48 \mathrm{~h}$ drug wash out. Raw data of $\mathrm{CI}$ and beating profile were obtained from the xCELLigence RTCA Cardio system for the assessment of the alterations in hPSC-CMs functional properties. In addition, to perform qRT-PCR analysis of genomic and miRNA biomarkers, ETP-treated hPSCCMs were harvested on day 2 with respective untreated controls. Our initial data show that single dose of $>30 \mu \mathrm{M}$ ETP had very high cytotoxicity towards hPSC-CMs and leads to drastic drop in the CI (Fig. 1b), whereas single dose of $<10 \mu \mathrm{M}$ ETP had no significant effect on CI or hPSC-CMs' functional properties. Interestingly, 30 and $15 \mu \mathrm{M}$ ETP caused irreversible increase in the beating rate of hPSC-CMs leading to alterations in the beating profile and arrhythmic beating (Fig. 1c, d). Furthermore, $10 \mu \mathrm{M}$ ETP also showed initial increase in beating rate and changes in beating profile, but hPSC-CMs were able to recover to basal levels after drug wash out. Similarly, we also observed alterations in the beating amplitude of hPSC-CMs due to ETP treatment (Fig. 1e). The level of extracellular lactate dehydrogenase (LDH) was used as an indicator of membrane damage and cell death (Chan et al. 2013). We observed significant and dose-dependent increase in extracellular LDH level postETP treatment, indicating membrane damage and stimulation of apoptotic cell death in hiPSC-CMs (Fig. 1f). Based on these findings, ETP at 10, 15, and $30 \mu \mathrm{M}$ was considered the most effective concentration and used for further cardiotoxicity assessments. The raw data from quadruplet experiment were acquired, analyzed, and represented as percent cytotoxicity with \pm SEM (Chan et al. 2013).

\section{The qRT-PCR based analysis of hiPSC-CMs treated with single high dose of etoposide identifies cluster of deregulated genomic and miRNA markers}

Applying wide genome microarrays, we recently reported array of 84 genes (Chaudhari et al. 2016b) and 14 miRNAs (Chaudhari et al. 2016a) as potential biomarkers for the assessment of drug-induced cardiotoxicity using hiPSCCMs. These biomarkers include 50 down- and 34 upregulated genes and 4 down- and 10 upregulated miRNAs. To explore the underlying mechanisms involved in ETPinduced cardiotoxicity, we performed qRT-PCR analysis of these biomarkers from hPSC-CMs treated with single high dose of $(10,15$, and $30 \mu \mathrm{M})$ ETP for $48 \mathrm{~h}$. Genes with at least 1.8 -fold changes were identified and grouped based on fold change values. Three groups of genes were identified; group-1 represents downregulated genes (Fig. 2a); group-2 represents upregulated genes (Fig. 2b); and group-3 represents genes with no change in fold expression values (Fig. 2c). Genes from group-1 and -2 were further sub-categorized in three clusters based on concentration of drug and fold change values (Fig. 2d). Altogether, out of 84 genes, 58 were deregulated consisting 25 down- and 33 upregulated genes. Fold change values of all the deregulated genes are shown in Supplementary table (1). To further investigate the biological functions and molecular pathway involvement of these genes, Database for Annotation, Visualization and Integrated Discovery (DAVID) was used for functional annotation and gene ontology (GO) clustering (Dennis et al. 2003). KEGG pathway and GO analysis showed that downregulated genes from group-1 were enriched in 4 KEGG pathways and GOs including muscle contraction, cardiac muscle contraction, calcium ion transport from endoplasmic reticulum to cytosol, and cytoskeleton organization (Table 1). Similarly, upregulated genes from group 2 were mainly enriched in 1 KEGG pathway and GOs like regulation of cell death, regulation of apoptotic process, programmed cell death, positive regulation of apoptotic process, and mitochondrion organization (Table 2). In addition, we also identified 5 out of 14 miRNAs; hsa-miR-486-3p, hsamiR-34c-5p, hsa-miR-4423-3p, hsa-miR-182-5p, and hsamiR-139-5p deregulated after ETP treatment (Fig. 2e). Fold change values of all the deregulated miRNAs are shown in Supplementary table (2). The hsa-miR-486-3p, hsa-miR182-5p, and hsa-miR-139-5p showed dose-dependent upregulation in fold expression value, whereas hsa-miR-34c-5p and hsa-miR-4423-3p remained twofold upregulated with no significant deviation in fold expression at all three test concentration. Target genes of each upregulated miRNA were then identified using the miRWalk 2.0 bioinformatics tool (Dweep et al. 2011) and matched with ETP-induced downregulated genes as we previously reported (Chaudhari et al. 2016a). Common genes were analyzed for the enrichment of GO and KEGG pathways using the DAVID. As shown in Supplementary data 1 , the downregulated genes were mainly enriched in GO terms such as the muscle contraction, regulation of heart contraction, actin filament-based process, and cardiac muscle cell action potential and KEGG pathways such as cardiac muscle contraction, arginine and proline metabolism, and hypertrophic cardiomyopathy (HCM). Taken together, these findings indicate that ETP induces cardiotoxicity mainly via cytoskeletal damage and activation of cell death via apoptosis and death receptor signaling in CMs. In addition, we also show that using combination of hiPSC-CMs and cardiotoxicity biomarkers, we can assess the cardiotoxicity of known or novel chemical entities.

\section{Etoposide induces cytoskeletal damage and sarcomeric deterioration in hiPSC-CMs}

The earliest morphological changes in iCell-CMs exposed to high concentrations of ETP were evident. Visible alterations in cell morphology, vacuolization within the sarcoplasm and alterations in cell size were observed after treatment (Fig. S1A). Even though the qRT-PCR data did not show any significant deregulation of cardiac cytoskeletal 
A

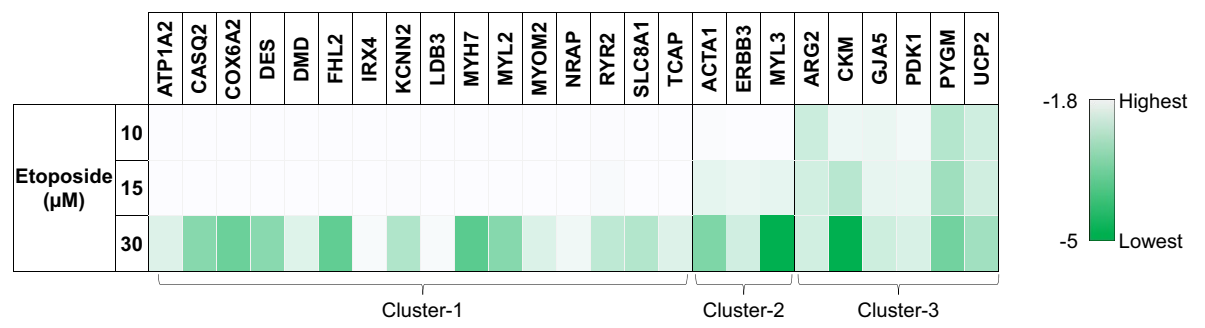

B

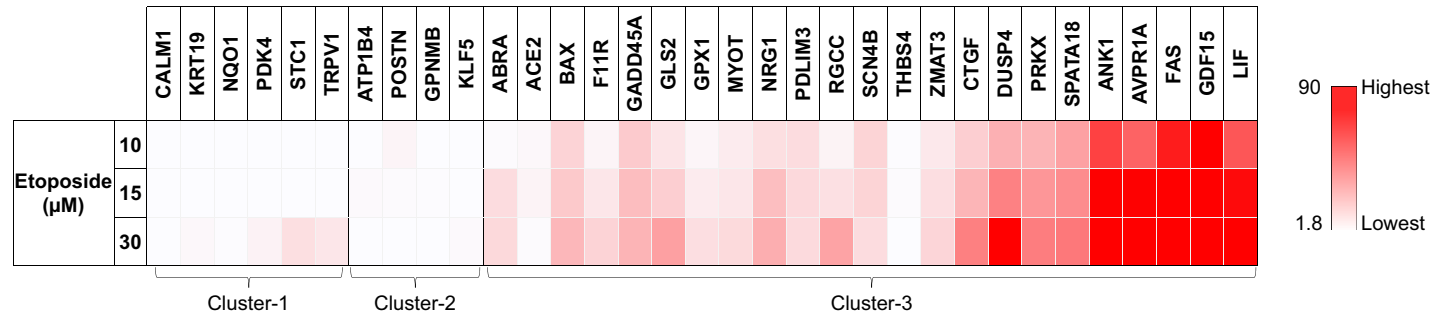

C

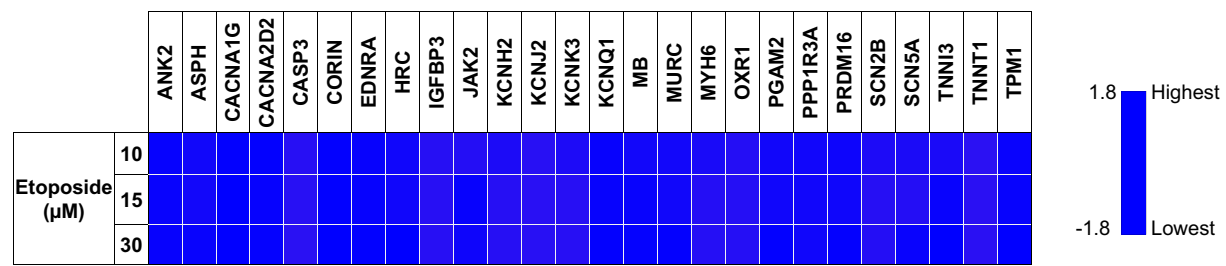

D
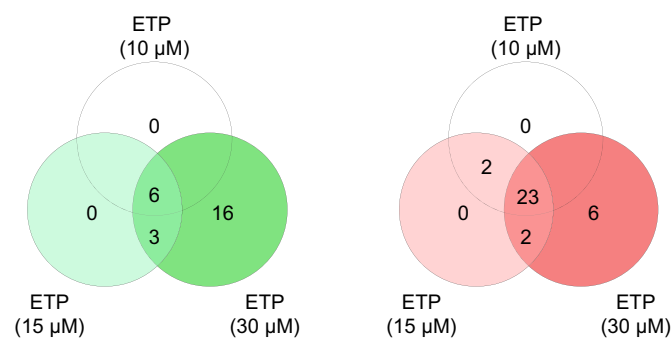

Fig. 2 Etoposide deregulates cluster of genomic and miRNA biomarkers in hiPSC-CMs. Heat maps showing ETP-induced deregulation of genomic and miRNA markers in CMs. a Represents genes downregulated after ETP treatment $(n=3$, fold change $\leq-1.8)$. b Represents genes upregulated after ETP treatment $(n=3$, fold

genes like MYH6, TNNI3, and TNNT1, downregulation in expression of MLY2, MYL3, MYH7, MYOM2, DES, and ACTA1 genes was observed after ETP treatment. In addition, ERBB3 and FHL2, which are important for normal cardiac development and play a crucial role in recovering cardiomyocytes from various types of cardiac insults (Erickson et al. 1997; Tran et al. 2016), were also found to be downregulated after ETP treatment. Many of these genes
$\mathbf{E}$

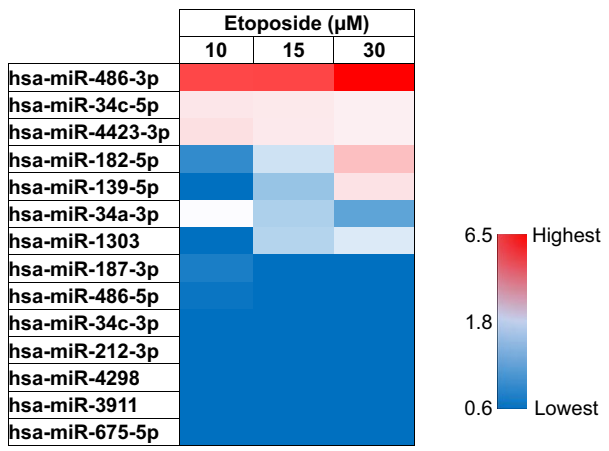

change $\geq 1.8$ ). c Represents genes with no significant change in expression level after ETP treatment $(n=3)$. d Venn diagram showing commonly deregulated genes in groups with different ETP concentrations $(n=3)$. e Represents deregulated miRNA markers $(n=3)$

belong to group-1/cluster-1 and group-1/cluster-2 indicating dose-dependent cytotoxicity of ETP. Using antibodies for anti-sarcomeric alpha actinin ( $\alpha$-Actinin) and cardiac troponin $\mathrm{T}(\mathrm{cTnT})$, we further investigated the effect of ETP exposure on cardiac sarcomere structures. Immunostaining revealed disorganizations in cardiac sarcomere structures in hPSC-CMs treated with ETP compared to that of untreated cells (Fig. 3a). Particularly, severe cytoskeletal deterioration 
Table 1 Significantly enriched GO categories and pathways by group-1 genes (downregulated genes after etoposide treatment)

\begin{tabular}{|c|c|c|c|}
\hline Components & Gene count & $p$ value & Representative genes \\
\hline \multicolumn{4}{|l|}{ GO terms: Group-1 } \\
\hline GO:0006936-muscle contraction & 15 & $7.00 \mathrm{E}-19$ & $\begin{array}{l}\text { SLC8A1, MYL2, ACTA1, TCAP, MYL3, MYH7, } \\
\text { ATP1A2, GJA5, DES, MYOM2, ARG2, DMD, } \\
\text { KCNN2, RYR2, CASQ2 }\end{array}$ \\
\hline GO:0060048—cardiac muscle contraction & 11 & $2.49 \mathrm{E}-16$ & $\begin{array}{l}\text { SLC8A1, MYL2, TCAP, MYL3, DMD, KCNN2, RYR2, } \\
\text { MYH7, ATP1A2, GJA5, CASQ2 }\end{array}$ \\
\hline GO:0060047-heart contraction & 11 & $3.52 \mathrm{E}-13$ & $\begin{array}{l}\text { SLC8A1, MYL2, TCAP, MYL3, DMD, KCNN2, RYR2, } \\
\text { MYH7, ATP1A2, GJA5, CASQ2 }\end{array}$ \\
\hline $\begin{array}{l}\text { GO:0086065-cell communication involved in cardiac } \\
\text { conduction }\end{array}$ & 6 & $4.47 \mathrm{E}-09$ & SLC8A1, KCNN2, RYR2, ATP1A2, GJA5, CASQ2 \\
\hline $\begin{array}{l}\text { GO: } 0010882 \text { - regulation of cardiac muscle contraction } \\
\text { by calcium ion signaling }\end{array}$ & 5 & $2.33 \mathrm{E}-08$ & SLC8A1, DMD, RYR2, ATP1A2, CASQ2 \\
\hline $\begin{array}{l}\text { GO: } 1903514 \text { - calcium ion transport from endoplasmic } \\
\text { reticulum to cytosol }\end{array}$ & 5 & $3.32 \mathrm{E}-08$ & SLC8A1, DMD, RYR2, ATP1A2, CASQ2 \\
\hline GO:0042391—regulation of membrane potential & 8 & $3.58 \mathrm{E}-07$ & $\begin{array}{l}\text { SLC8A1, UCP2, DMD, KCNN2, RYR2, ATP1A2, GJA5, } \\
\text { CASQ2 }\end{array}$ \\
\hline GO:0007010—cytoskeleton organization & 7 & 0.0036 & DES, MYL2, TCAP, ACTA1, DMD, LDB3, CASQ2 \\
\hline GO:0015992—proton transport & 3 & 0.0184 & UCP2, COX6A2, ATP1A2 \\
\hline \multicolumn{4}{|l|}{ KEGG pathways: Group-1 } \\
\hline hsa04260:Cardiac muscle contraction & 6 & $3.63 \mathrm{E}-07$ & MYL2, MYL3, COX6A2, RYR2, MYH7, ATP1A2 \\
\hline hsa05410:Hypertrophic cardiomyopathy (HCM) & 5 & $1.87 \mathrm{E}-05$ & DES, MYL2, MYL3, DMD, RYR2 \\
\hline hsa05414:Dilated cardiomyopathy & 5 & $2.51 \mathrm{E}-05$ & DES, MYL2, MYL3, DMD, RYR2 \\
\hline $\begin{array}{l}\text { hsa05412:Arrhythmogenic right ventricular cardiomyo- } \\
\text { pathy (ARVC) }\end{array}$ & 3 & 0.010025 & DES, DMD, RYR2 \\
\hline
\end{tabular}

was observed at $30 \mu \mathrm{M}$ ETP (Fig. 3b). Subsequent transmission electron microscopy analysis also confirmed the membrane and cytoskeletal damage including irregularities in cardiac myofibrillar arrangements in ETP-treated hPSCCMs (Fig. 3c). Collectively, these results show that ETP exposure induces cell membrane damage and disorganization of cardiac sarcomere structures.

\section{Etoposide causes alterations in calcium handling in hiPSC-CMs}

Calcium $\left(\mathrm{Ca}^{2+}\right)$ is the critical intermediary in the process of cardiac EC-coupling, connecting hPSC-CMs depolarization with mechanical contraction. In addition to opening cardiac type ryanodine receptors (RyR2) via the mechanism of $\mathrm{Ca}^{2+}$-induced $\mathrm{Ca}^{2+}$ release (CICR), $\mathrm{Ca}^{2+}$ is also critical in the regulation of several other important aspects of hPSC-CMs biological processes such gene transcription. We found several genes, among them CALM1, SLC8A1, DMD, RYR2, ATP1A2, CASQ2, and ERBB3, which play important roles in cardiac $\mathrm{Ca}^{2+}$ handling, to be deregulated in hPSC-CMs after ETP treatment. To investigate the effect of ETP on cardiac $\mathrm{Ca}^{2+}$ handling in greater detail, we recorded and analyzed spontaneous $\mathrm{Ca}^{2+}$ transients in hPSCCMs loaded with the $\mathrm{Ca}^{2+}$ indicator, Rhod-2 AM (Fig. 4a). ETP had concentration dependent, partially contrary effects on the $\mathrm{Ca}^{2+}$ transient amplitude and on the kinetics. The hiPSC-CMs treated with ETP at 10 and $15 \mu \mathrm{M}$ showed a higher $\mathrm{Ca}^{2+}$ transient amplitude (Fig. $4 \mathrm{~b}, F_{\text {max }} / F_{0}$ ), a slowing in reaching the $\left[\mathrm{Ca}^{2+}\right]$ peak (Fig. $4 \mathrm{~b}, \mathrm{TTP}$ ) as well a prolonged presence of $\mathrm{Ca}^{2+}$ in the sarcoplasm (Fig. 4b, T90\% and FWHM). In contrast, $30 \mu \mathrm{M}$ ETP caused an acceleration of the ascending phase of the transient (Fig. 4b, TTP and $\left.\Delta F / \Delta T_{\max }\right)$. The kinetics of $\mathrm{Ca}^{2+}$ cycling in hPSC-CMs are determined in large part by the complex interplay between the type 2 ryanodine receptor (RyR2), the cardiac dihydropyridine receptor (DHPR), and the SERCA2a Ca ${ }^{2+}$ pump. Subtle alterations in these components through mutation, dysregulation or drug toxicity can have profound effects on the function of CMs. Our data show that ETP exposure could indeed cause alterations in the function of one or more of these critical $\mathrm{Ca}^{2+}$ cycling components, which may lead to the observed altered $\mathrm{Ca}^{2+}$ transients and which ultimately will affect the normal functionality of CMs.

\section{Etoposide upregulates apoptosis signaling in hiPSC-CMs}

Like many other Topoisomerase II inhibitors, ETP has been reported to induce apoptosis in thymocytes, BAF3 cell line and other cancer lines; this induction of apoptosis mainly has been linked to DNA fragmentation originated by 
Table 2 Significantly enriched GO categories and pathways by group-2 genes (upregulated genes after etoposide treatment)

\begin{tabular}{|c|c|c|c|}
\hline Components & Gene count & $p$ value & Representative genes \\
\hline \multicolumn{4}{|l|}{ GO terms: Group-2 } \\
\hline GO:0010941—regulation of cell death & 14 & $1.61 \mathrm{E}-06$ & $\begin{array}{l}\text { TRPV1, ZMAT3, PDK4, GLS2, GPX1, CTGF, BAX, } \\
\text { RGCC, FAS, NQO1, GPNMB, GDF15, NRG1, } \\
\text { GADD45A }\end{array}$ \\
\hline GO:0042981—regulation of apoptotic process & 13 & $5.02 \mathrm{E}-06$ & $\begin{array}{l}\text { GLS2, GPX1, TRPV1, CTGF, ZMAT3, BAX, RGCC, } \\
\text { PDK4, FAS, GDF15, NRG1, NQO1, GADD45A }\end{array}$ \\
\hline GO:0033554—cellular response to stress & 14 & $9.76 \mathrm{E}-06$ & $\begin{array}{l}\text { GPX1, TRPV1, CTGF, ZMAT3, BAX, SPATA18, RGCC, } \\
\text { PDK4, AVPR1A, STC1, FAS, NQO1, GADD45A, } \\
\text { THBS4 }\end{array}$ \\
\hline GO:0043065-positive regulation of apoptotic process & 8 & $8.22 \mathrm{E}-05$ & $\begin{array}{l}\text { TRPV1, CTGF, ZMAT3, BAX, RGCC, FAS, NQO1, } \\
\text { GADD45A }\end{array}$ \\
\hline GO:0012501—programmed cell death & 13 & $8.51 \mathrm{E}-05$ & $\begin{array}{l}\text { GLS2, GPX1, TRPV1, CTGF, ZMAT3, BAX, RGCC, } \\
\text { PDK4, FAS, GDF15, NRG1, NQO1, GADD45A }\end{array}$ \\
\hline GO:0043408—regulation of MAPK cascade & 8 & $2.36 \mathrm{E}-04$ & $\begin{array}{l}\text { LIF, DUSP4, CTGF, FAS, GPNMB, GDF15, NRG1, } \\
\text { GADD45A }\end{array}$ \\
\hline $\begin{array}{l}\text { GO:2001239-regulation of extrinsic apoptotic signal- } \\
\text { ing pathway in absence of ligand }\end{array}$ & 3 & 0.0042 & BAX, FAS, NRG1 \\
\hline GO:0097191—extrinsic apoptotic signaling pathway & 4 & 0.0088 & GPX1, BAX, FAS, NRG1 \\
\hline GO:0010821—regulation of mitochondrion organization & 4 & 0.0093 & GLS2, GPX1, BAX, NRG1 \\
\hline $\begin{array}{l}\text { GO:0008625—extrinsic apoptotic signaling pathway via } \\
\text { death domain receptors }\end{array}$ & 3 & 0.0099 & GPX1, BAX, FAS \\
\hline $\begin{array}{l}\text { GO:0042770—signal transduction in response to DNA } \\
\text { damage }\end{array}$ & 3 & 0.0215 & BAX, RGCC, GADD45A \\
\hline GO:0007005—mitochondrion organization & 5 & 0.0344 & GLS2, GPX1, BAX, SPATA18, NRG1 \\
\hline \multicolumn{4}{|l|}{ KEGG pathways: Group-2 } \\
\hline hsa04115:p53 signaling pathway & 4 & $7.56 \mathrm{E}-04$ & ZMAT3, BAX, FAS, GADD45A \\
\hline
\end{tabular}

inhibition of Topoisomerase II (Onishi et al. 1993; Walker et al. 1991). In addition, in recent study upregulation FAS has been reported as a critical mechanism involved in DOXinduced cardiotoxicity (Zhao and Zhang 2017). In accordance with these findings, our qRT-PCR data show that ETP treatment leads to upregulation of genes involved in apoptosis and death receptor signaling like BAX, FAS, RGCC, GLS2, GPX1, ZMAT3, and GADD45A, although we did not observed any significant upregulation in caspase 3 (CASP3) mRNA levels after ETP treatment. To further confirm the activation of apoptosis, we stained ETP-treated hPSC-CMs with Phosphatidylserine (PS) sensor, apopxin and 7-aminoactinomycin D (7-AAD), for late stage apoptosis and necrosis. Confocal images clearly show activation of apoptosis after $48 \mathrm{~h}$ of ETP treatment. Notable, increase in number of red stained nuclei was observed in hPSC-CMs treated with $30 \mu \mathrm{M}$ ETP indicating severe membrane damage and late stage apoptosis and necrosis (Fig. 5a). To ascertain the role of apoptosis signaling in ETP-induced cardiotoxicity, we then performed RTCA experiments, where hPSC-CMs were treated with ETP in the presence of Pifithrin- $\alpha$ (an inhibitor of p53 transcriptional activity) and real-time data of hPSC-CMs CI and beating rate were obtained from the xCELLigence RTCA Cardio system. Pifithrin- $\alpha$ has been shown to protect hPSC-CMs against DOX-induced apoptosis in mouse heart (Liu et al. 2004). It inhibits apoptosis and attenuates DOX-induced elevation in Bax and MDM2 protein levels. Our RTCA data show that Pifithrin- $\alpha$ did not prevent ETP-induced cytotoxicity (Fig. 5b) but interestingly it did prevent the ETP-induced increase in beating rate in hiPSC-CMs (Fig. 5c). Pifithrin- $\alpha$ alone causes slight increase in beating rate (Fig. S2A). Altogether, these data suggest that apoptosis signaling might be the crucial player in ETP-induced cardiotoxicity and Pifithrin- $\alpha$ contributed to rescue the hPSC-CMs from ETP-induced cardiotoxicity.

\section{Etoposide induces mitochondrial damage, increase of ROS production and loss of mitochondrial membrane potential in hiPSC-CMs}

Measurements of intracellular ATP levels from ETPtreated hiPSC-CMs showed drop in the ATP levels indicating inhibition of ATP production and altered mitochondrial bioenergetics function (Fig. S1B). To further assess the mitochondrial quality and structure we performed transmission electron microscopy and JC-1 staining of ETP-treated CMs. TEM imaging showed intact, elongated mitochondria with clear cristae in untreated 
Fig. 3 Etoposide induces cytoskeletal disorganization in hiPSC-CMs. a, b Immunostaining of cardiac sarcomeric $\alpha$-actinin ( $\alpha$-Actinin) and cardiac troponin $\mathrm{T}$ (cTnT) in untreated and ETP-treated hiPSC-CMs. Nuclei are stained with DAPI. Scale bar represents 50, $5 \mu \mathrm{m}$. c Represents TEM images of untreated and ETPtreated hiPSC-CMs. Scale bar represents $5000 \mathrm{~nm}$. (1, Z-line; 2, sarcomere; 3 , mitochondria; 4 , myofibril bundles)
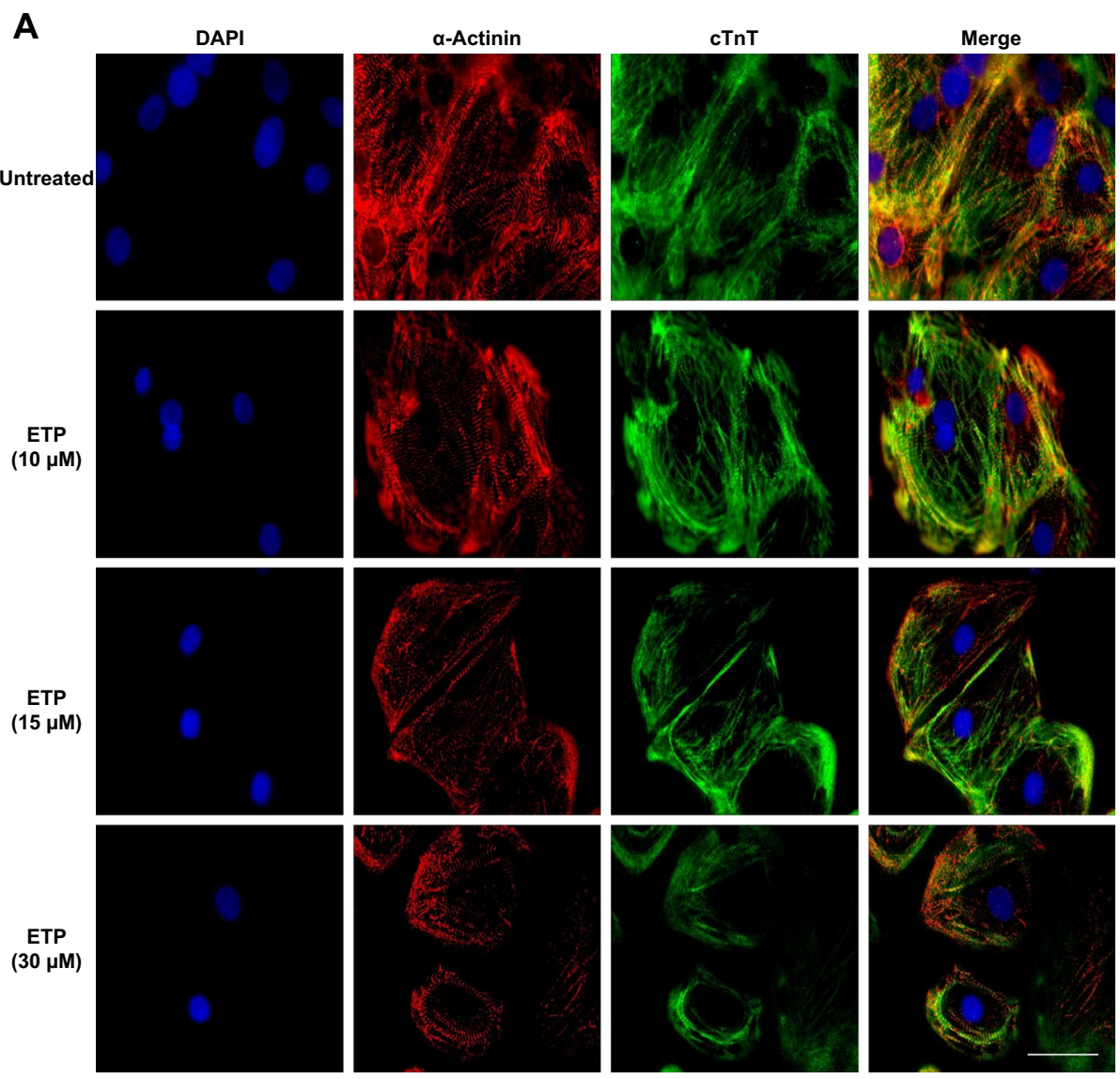

B

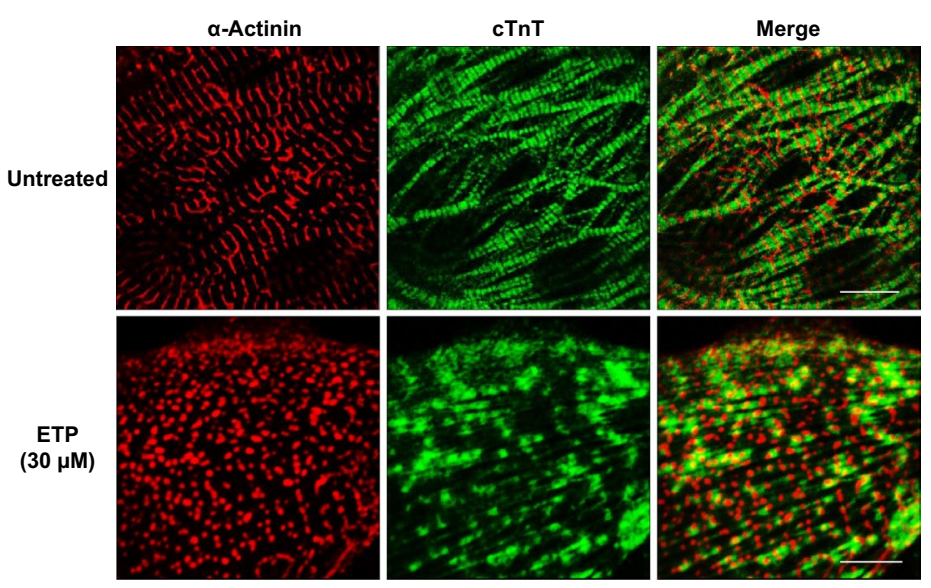

C

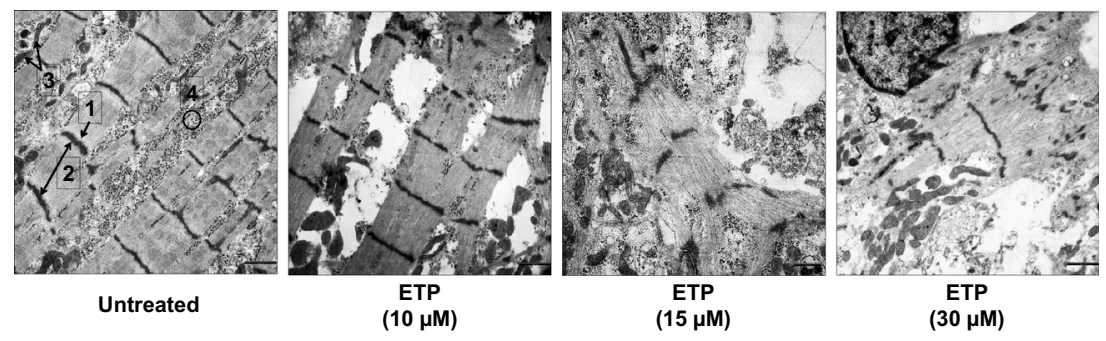




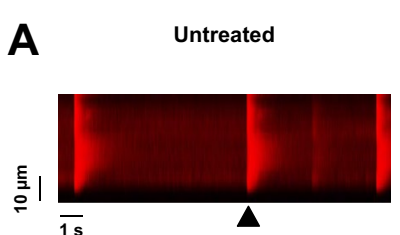

ETP

$(10 \mu \mathrm{M})$

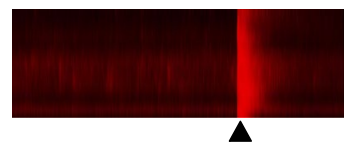

$\triangle$
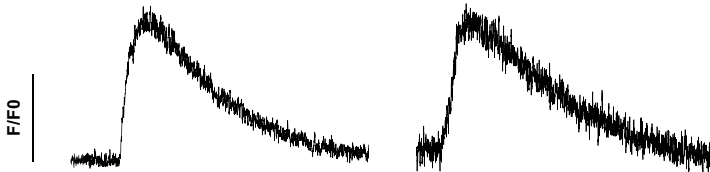

B

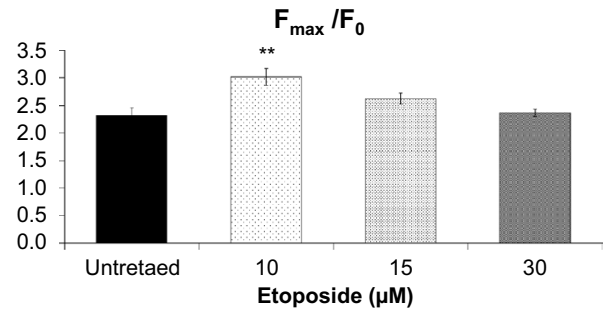

$\mathrm{T} 90 \%$
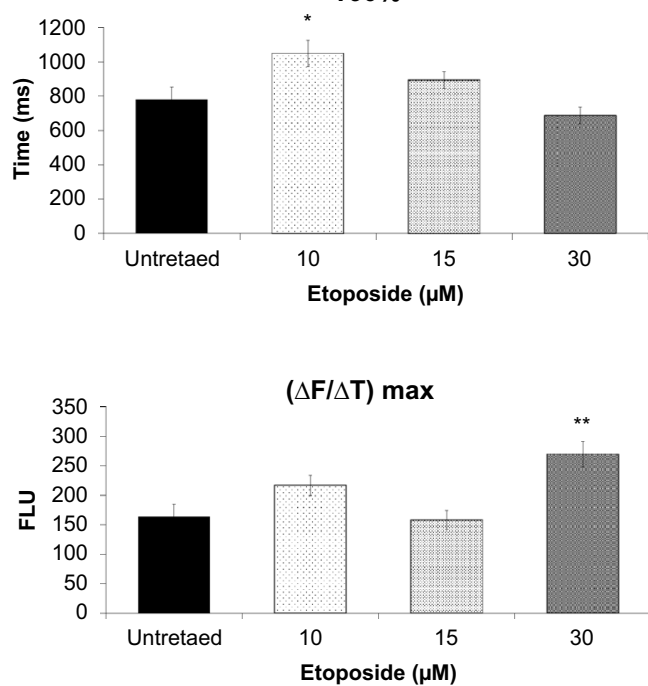

Fig. 4 Etoposide causes alterations in calcium handling in hiPSCCMs. a Confocal line-scan images showing changes in intracellular $\left[\mathrm{Ca}^{2+}\right]_{i}$ in a Rhod-2, AM loaded hiPSC-CM. The images show alterations in spontaneous whole-cell $\mathrm{Ca}^{2+}$ transients in response to ETP treatment (upper panel). Scale bar represents, time $-1 \mathrm{~s}$ and distance $-10 \mu \mathrm{m}$. Representative tracings of spontaneous $\mathrm{Ca}^{2+}$ transients (black arrow head) in hiPSC-CMs from untreated and ETP-treated

hPSC-CMs, whereas damage to mitochondrial membrane and mitochondrial fragmentation was observed in ETPtreated CMs. As reported, ETP at $30 \mu \mathrm{M}$ showed severe damage and fragmentation of mitochondria compared to that of an untreated cells (Fig. 6a). This mitochondrial damage can cause an imbalance between reactive oxygen species (ROS) production and removal, resulting in net

\section{ETP}

$(15 \mu \mathrm{M})$
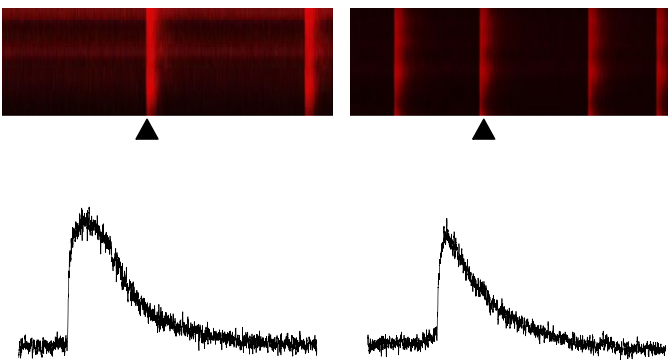

ETP

$(30 \mu \mathrm{M})$
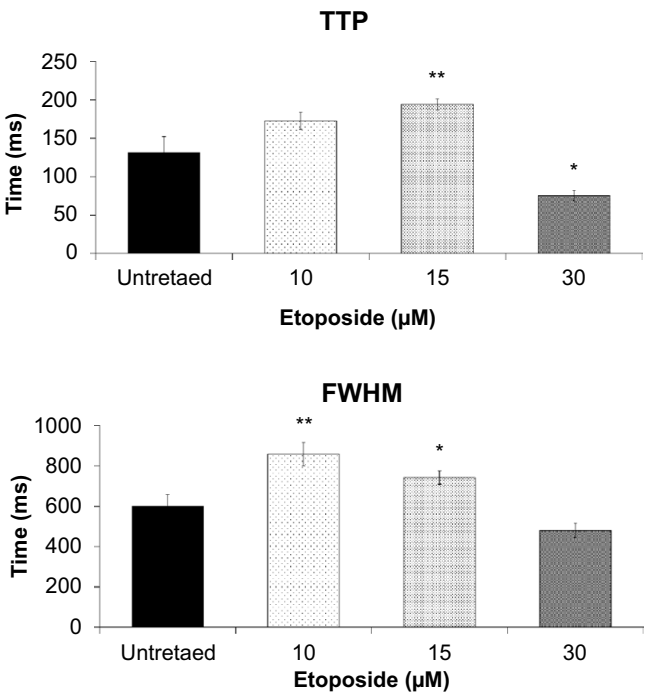

Fo

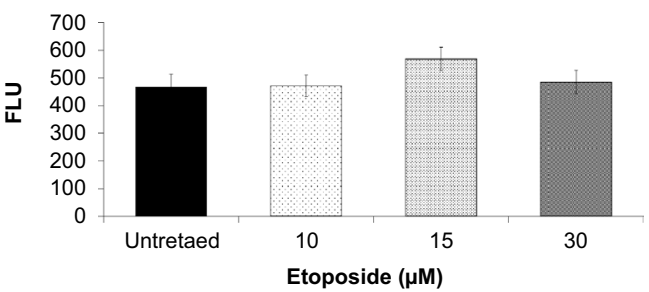

groups (lower panel). b Graphs representing $\mathrm{Ca}^{2+}$ transient parameters measured from hiPSC-CMs treated with ETP. $F / F_{0}, \mathrm{Ca}^{2+}$ transient amplitude where $F_{0}$ is the averaged background-corrected resting fluorescence intensity; TTP, time-to-peak; T90\%, 90\% recovery of $F_{\max } ;[\Delta F / \Delta T]_{\max }$, the maximum steepness; FWHM, full-width at half-maximum. $(n=25$, error bars represent $\pm \operatorname{SEM})\left(t\right.$ test, ${ }^{*} p \leq 0.05$, $* * p \leq 0.01)$

increase in ROS production. To evaluate ROS production after ETP treatment we used Dihydroethidium (DHE) staining. Confocal imaging revealed that ETP-treated hPSC-CMs had dose-dependent increase in nuclear DHE staining suggesting net increase in ROS production in a dose-dependent manner (Fig. 6b). The abnormal enlargements in cell and nuclear size were previously correlated 
Fig. 5 Etoposide upregulates apoptosis signaling in hiPSCCMs. a Fluorescent images showing live cells (blue), apoptotic cells (green), and necrotic cells (red) in hiPSCCMs after ETP treatment for $48 \mathrm{~h}$. Scale bar represents $10 \mu \mathrm{m}$. b, c hiPSC-CMs were co-treated with ETP and $10 \mu \mathrm{M}$ Pifithrin- $\alpha$ for 48 h. Real-time data of hPSC-CMs cell index and beating rate were obtained using xCELLigence RTCA system. Representative graphs display normalized CI and \% beating rate values, respectively, showing Pifithrin- $\alpha$ had significant effect in preventing ETPinduced cardiotoxicity $(n=3$, error bars represent \pm SEM) $(t$ test, $* p \leq 0.05, * * * p \leq 0.001)$ (see also Fig. S2A). (Color figure online)

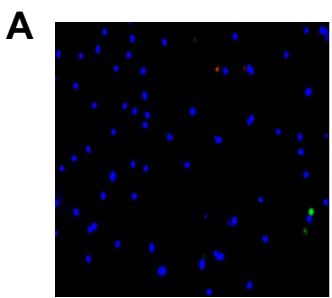

Untreated

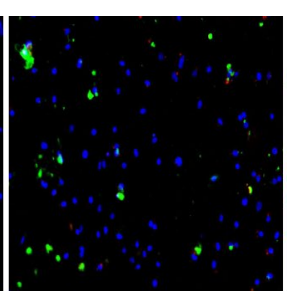

ETP

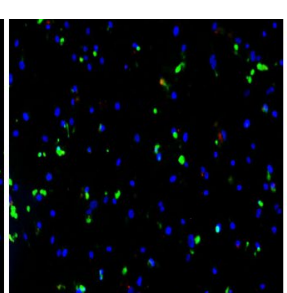

ETP

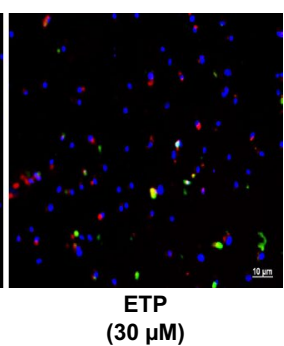

B

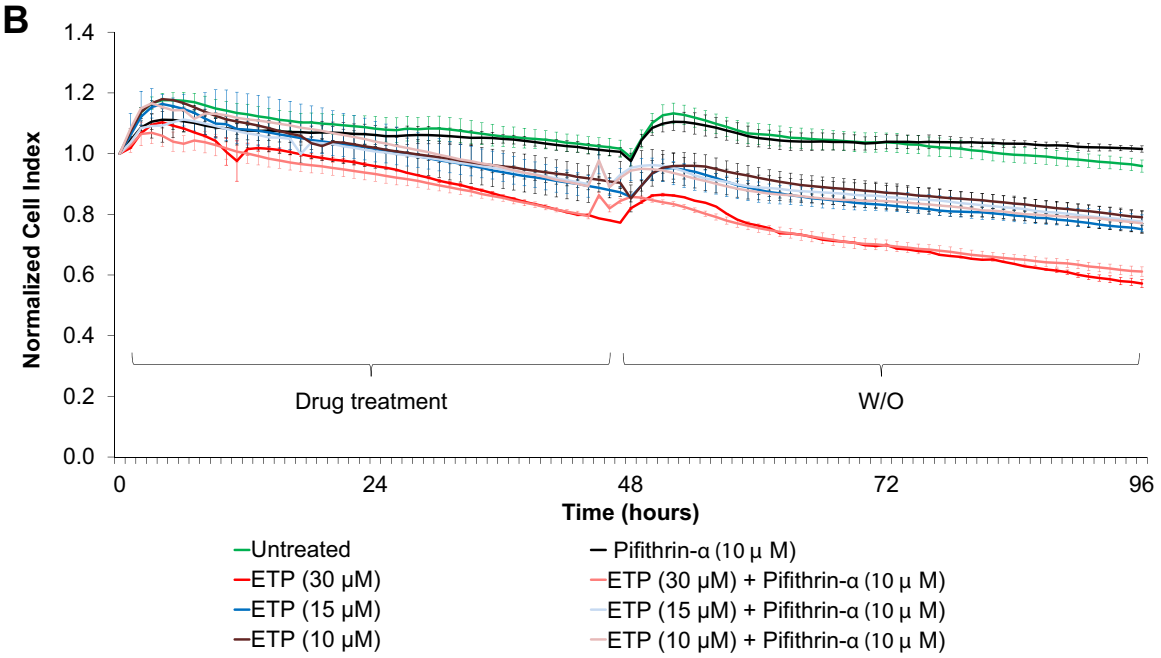

C

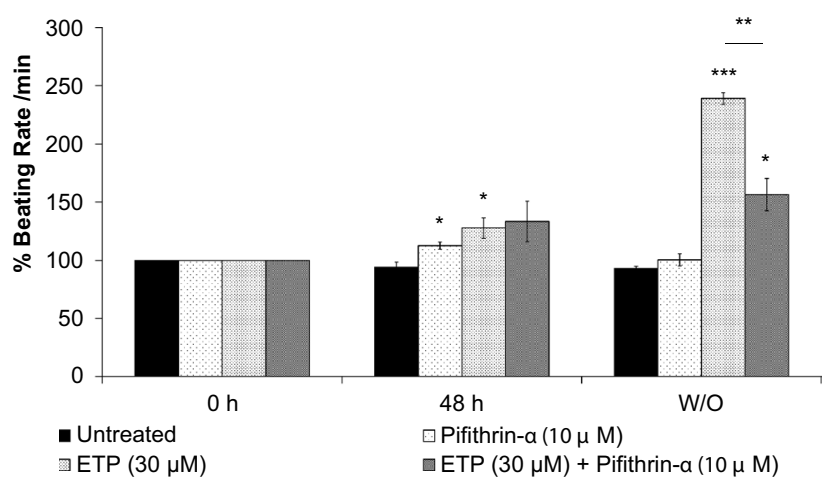

with anti-cancer drugs induced side effects (Kang et al. 2011). Nuclear staining with DAPI showed increased nuclear size in hiPSC-CMs treated with ETP indicating DNA damage and chromosomal abnormalities which could result in genomic instability. This DNA damage was also confirmed by upregulation in DNA damage response genes like RGCC, ZMAT3 and GADD45A in ETP-treated CMs. It has been shown that SPATA18 interacts with BH3 domain of NIX in ROS dependent manner and induces vacuole-like structures within mitochondria which engulf and degrade the unhealthy mitochondria (Kitamura et al. 2011). Our qRT-PCR data showed significant upregulation in SPATA18 and key genes responsible for regulation of
ROS generation like NQO1, GLS2, and GPX1 and, therefore, indicate increased ROS production and mitochondrial damage in ETP-treated CMs. We next investigated the effect of ETP on mitochondrial membrane potential $(m \Delta \psi)$ using JC-1 staining. Healthy mitochondria give out red fluorescence because of J aggregates of JC-1 dye and dead/apoptotic cells show green fluorescence due to lack of mitochondrial membrane potential (Mathur et al. 2000). Confocal imaging shows that like DOX (positive control) ETP exposure leads to significant loss of mitochondrial membrane potential in a dose-dependent manner compared to that of an untreated cells (Fig. 6c). Altogether, these findings indicate that increased mitochondrial damage and 
Fig. 6 Etoposide induces mitochondrial damage, increased ROS production and loss of mitochondrial membrane potential $(m \Delta \psi)$ in hiPSCCMs. a TEM images showing morphological alterations in mitochondrial membrane and cristae structures in hiPSC-CMs treated with ETP compared to untreated CMs. Scale bar represents $500 \mathrm{~nm}$. b Fluorescent images showing increase DHE staining in hiPSC-CMs treated with ETP compared to untreated $\mathrm{CMs}$, indicating net increase in ROS production. Nuclei are stained with DAPI which also show increased nuclear size after ETP treatment. Scale bar represents $20 \mu \mathrm{m}$. c Determination of mitochondrial membrane potential through JC-1 staining. Mitochondria of hiPSC-CMs after incubation with JC-1 dye, illustrating the heterogeneity in mitochondrial membrane potential in the same cell. The mitochondria membrane potential was found to be interrupted after DOX (positive control) and ETP treatment, as evidenced by reduction in the JC-1 red fluorescence signal. In addition hPSC-CMs treated with $30 \mu \mathrm{m}$ ETP for $48 \mathrm{~h}$ showed increased mitochondrial fragmentation further supporting the TEM data. Scale bar represents $20 \mu \mathrm{m}$ (upper panel), $5 \mu \mathrm{m}$ (lower panel)

A

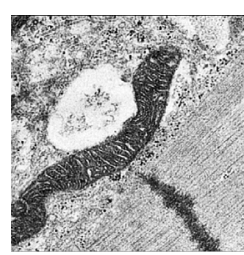

Untreated

B
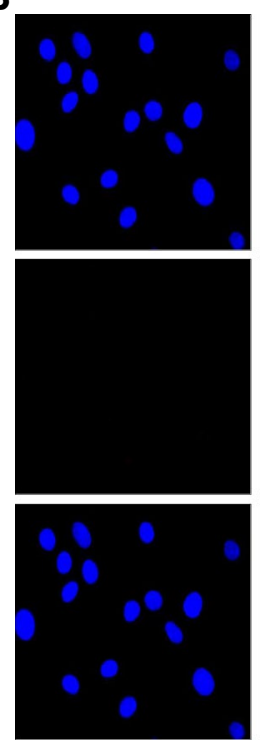

Untreated

C
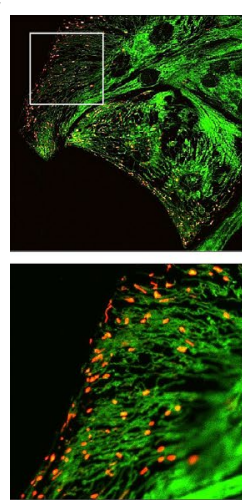

Untreated

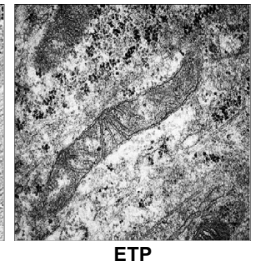

$(10 \mu \mathrm{M})$
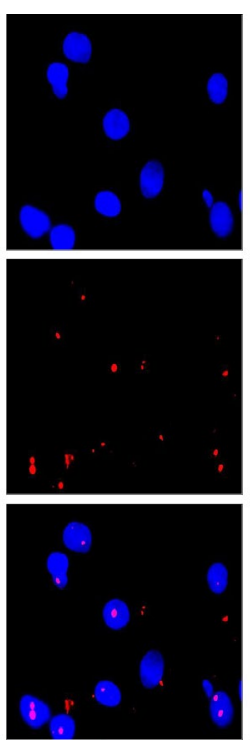

ETP
$(10 \mu \mathrm{M})$

$(10 \mu \mathrm{M})$
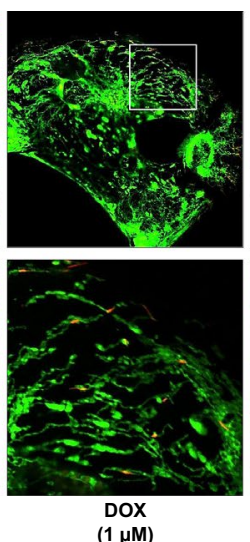
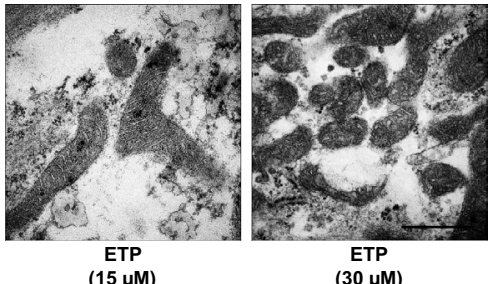

(30 $\mu \mathrm{M})$
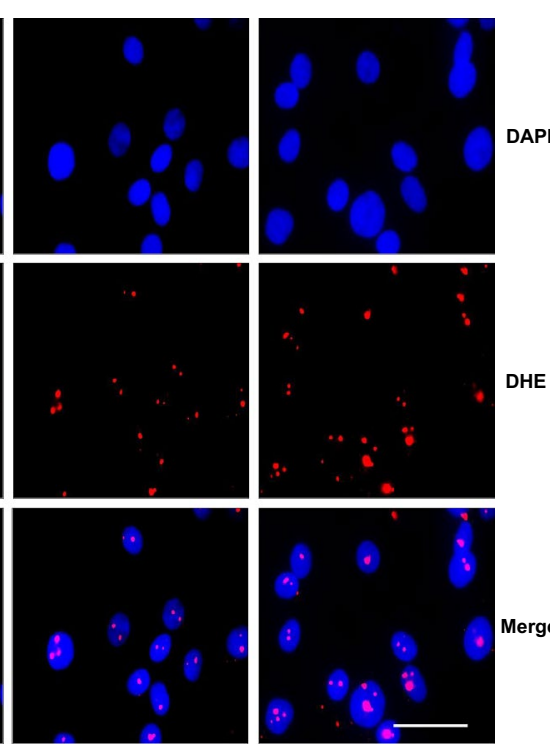

ETP

(15 $\mu \mathrm{M})$
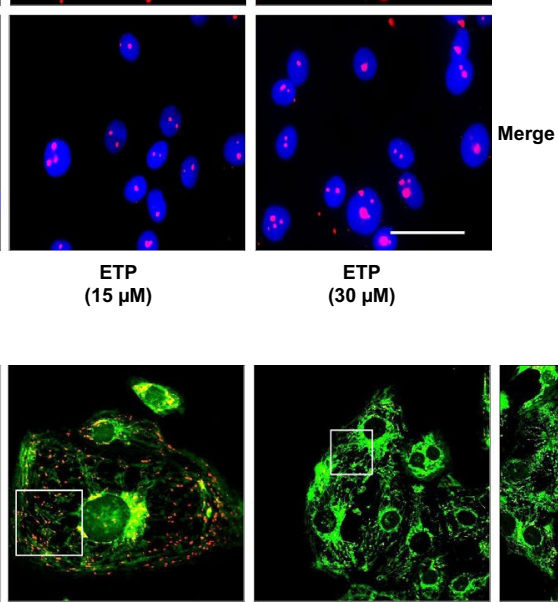

ETP
$(30 \mu \mathrm{M})$
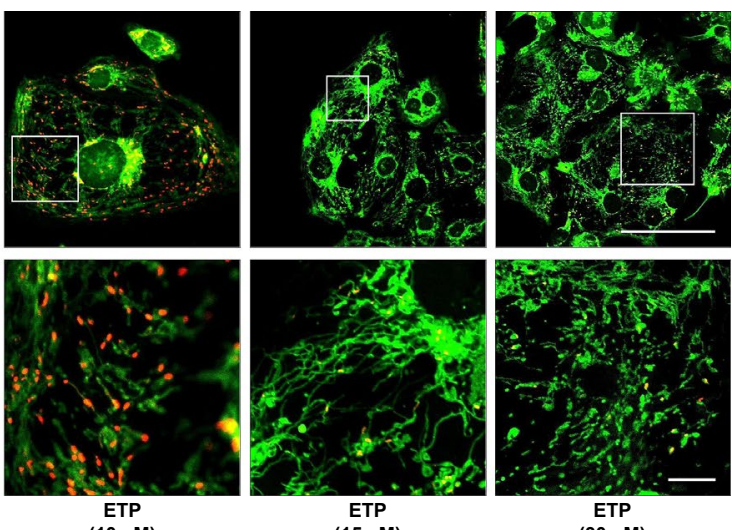

$(15 \mu \mathrm{M})$

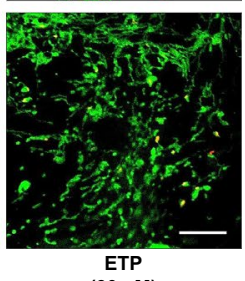

$(30 \mu \mathrm{M})$ activation of p53-SPATA18 pathway to repair or eliminate damaged mitochondria in hiPSC-CMs after ETP treatment.

\section{Ferroptosis inhibitor, Liproxstatin-1 helps to improve cardiomyocytes functional properties after etoposide treatment}

Ferroptosis is a form of an iron-dependent non-apoptotic cell death (Dixon et al. 2012). It is characterized by increased ROS production and morphologically by mitochondrial deformities. Smaller than normal mitochondria with reduced/vanishing mitochondrial cristae indicated likely activation of ferroptosis in ETP-treated hiPSC-CMs, this is further supported by the increased ROS production (Xie et al. 2016), p53 upregulation and GLS2 upregulation (Gao et al. 2015). To evaluate whether ferroptosis plays a role in ETP-induced cardiotoxicity, we simultaneously treated hPSC-CMs with ETP and liproxstatin-1 (a ferroptosis inhibitor). The RTCA analysis showed that the treatment of liproxstatin-1 did not show significant improvement in hPSC-CMs 
viability during and after ETP treatment (Fig. 7a). However, during wash out, it significantly promoted hPSC-CMs recovery from increase in beating rate induced by ETP (Fig. 7b). In addition, at lower ETP concentrations, liproxstatin-1 treated hPSC-CMs were able to fully recover from ETPinduced alterations as seen by stabilized beating rate (Fig. S2B). These data suggest that ferroptosis might be involved in ETP-induced cardiotoxicity.

\section{Discussion}

Etoposide is a DNA topoisomerase II (TOP II) inhibitor that has significant activity against wide spectrum human cancers such as, small-cell lung cancer, non-Hodgkin's lymphoma, stomach cancer and ovarian cancer (Hansen et al. 1977; Jiang et al. 2016; Klumpp et al. 1993). ETP inhibit TOP II- $\alpha$ and TOP II- $\beta$ isoforms, thereby leading to genotoxicity and cell death. It has been in clinical use for more than two decades; however, clinical application of ETP are limited due to its side effects on organs like kidney and heart. Although ETP-induced arrhythmia are very rare (Kridis et al. 2013), however, it has been reported to induce atrial arrhythmia and infarction especially when combined with cytotoxic drug like cisplatin (Kucharz et al. 2016; Petrella et al. 1989). In addition, several case studies also reported myocardial infarction and vasospastic angina in ETP-treated patients (Schwarzer et al. 1991; Yano and Shimada 1996). Although the underlying mechanisms for ETP-induced cardiotoxicity and alterations in cardiac function remains to be fully explored, the previous studies on DOX-induced cardiotoxicity points-to the inhibition of TOP II- $\beta$ by ETP as one of the major contributing factor.

We found that single high dose of ETP induces cytotoxicity leading to cell death and arrhythmia indicated by irreversible increase in hPSC-CMs beating rate. Current methods for evaluation of cardiotoxicity rely on studies performed using cell line overexpressing cardiac ion channels, especially the human ether-a-go-go (hERG) channel or animal models, especially mice models. Yet, cardiovascular toxicity remains a major contributing factor for postapproval withdrawal of medicines (Ferri et al. 2013). Limitations with current cardiotoxicity models demand urgent need to develop human-relevant in vitro and in vivo cardiotoxicity models. In the present study, we have established an in vitro cardiotoxicity methodology for the monitoring of early as well as chronic cardiotoxicity events associated with ETP at the cellular, functional and genomic level. In accordance
Fig. 7 Liproxstatin-1 improves cardiomyocytes functional properties after etoposide treatment. The hiPSC-CMs were cotreated with ETP and $200 \mathrm{nM}$ Liproxstatin-1 (ferroptosis inhibitor) for $48 \mathrm{~h}$. Real-time data of hPSC-CMs cell index, beating rate, and beating amplitude were obtained using xCELLigence RTCA system. Representative graphs display a normalized $\mathbf{C I}$ and $\mathbf{b} \%$ beating rate values, respectively, showing even though Liproxstatin-1 had no significant effect in preventing ETP-induced cytotoxicity; it significantly improved hPSC-CMs ability to recover from ETP-induced alterations in beating rate and beating amplitude $(n=3$, error bars represent \pm SEM) $\left(t\right.$ test, ${ }^{*} p \leq 0.05$, $* * p \leq 0.01, * * * p \leq 0.001$ ) (see also Fig. S3A)
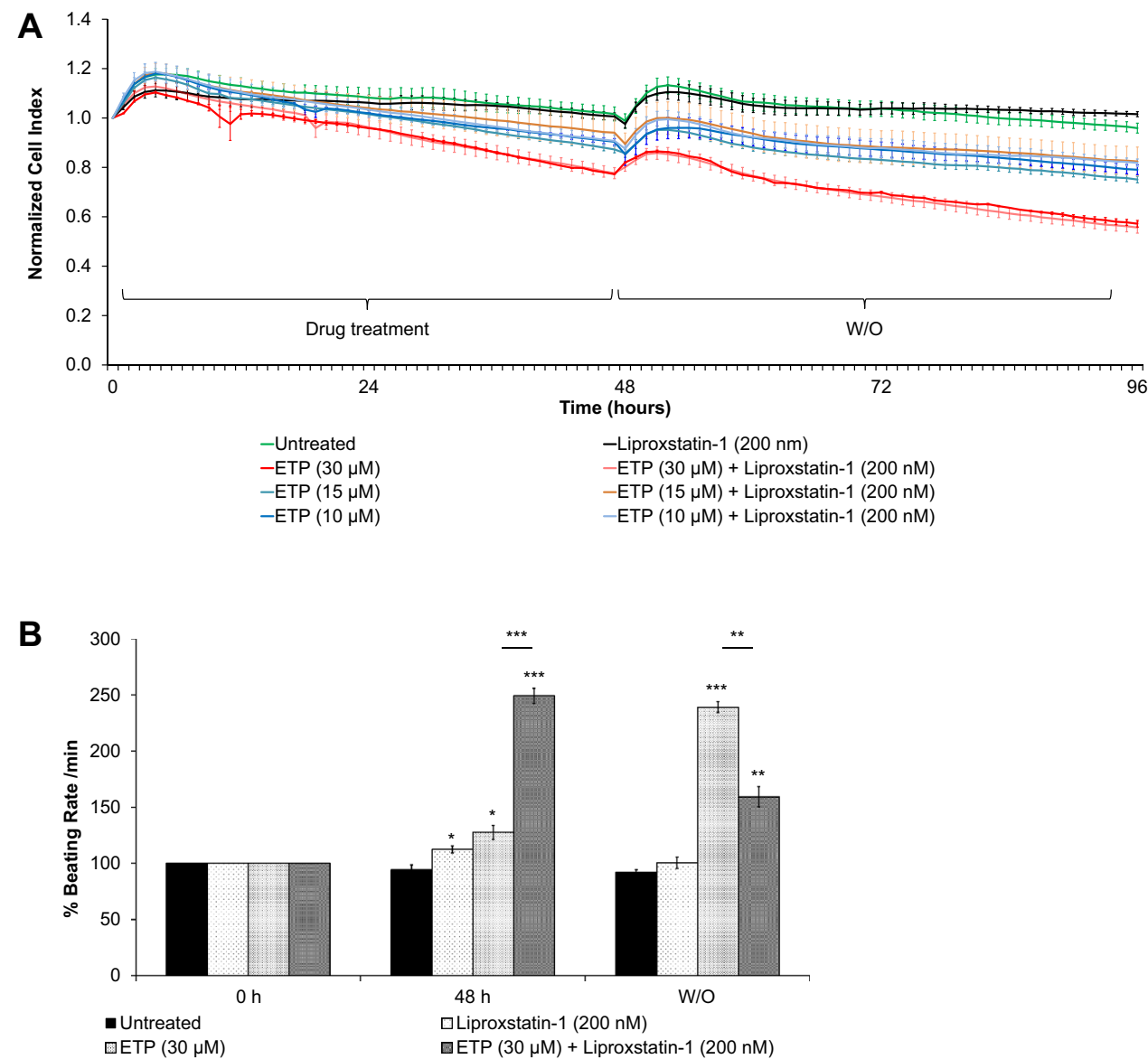
with the previous studies, we found increased expression levels of ACE2, DUSP4, LIF and NRG1 in hiPSC-CMs treated with ETP making them suitable candidate for in vitro cardiotoxicity markers (Choi et al. 2012; Goulter et al. 2004; Jougasaki et al. 2003; Yan and Morgan 2011). GDF15, a stress-responsive transforming growth factor-beta-related cytokine, have been reported as a biomarker in cardiovascular disease as well as a strong prognosticator of heart failure (Kempf et al. 2007; Kempf and Wollert 2009). We also found GDF15 highly upregulated in hiPSC-CMs after ETP treatment. Altogether, these findings suggest that the gene expression profiles observed using our in vitro system are very similar to those observed in patients with heart conditions and therefore offer human-relevant model which can be used as an effective alternative for costly and time consuming animal studies.

In addition we also identified 58 deregulated genes consisting 33 upregulated and 25 downregulated genes in hPSC-CMs after ETP treatment. GO and KEGG pathway analysis confirmed most upregulated genes are enriched in GO categories like positive regulation of apoptotic process, regulation of cell death, and mitochondria organization, whereas most downregulated genes were enriched in GO categories like cytoskeletal organization, muscle contraction and $\mathrm{Ca}^{2+}$ ion homeostasis. Moreover we also found upregulation in 5 miRNAs (has-miR-486-3p, has-miR-34c-5p, has-miR-4423-3p, has-miR-182-5p, and has-miR-139-5p) which play role in muscle contraction, Arginine and proline metabolism and Hypertrophic cardiomyopathy (HCM). Immunostaining and Transmission Electron Microscopy also confirmed the cytoskeletal and mitochondrial damage in hPSC-CMs treated with ETP. Using xCELLigence Real-Time Cell Analyser (RTCA), we show that apoptosis inhibitor, Pifithrin- $\alpha$, protect hPSC-CMs from ETP-induced cardiotoxicity, whereas hPSC-CMs treated with ferroptosis inhibitor, Liproxstatin-1, showed significant recovery during wash out period. Altogether, these observations show that ETP on one hand induces cytoskeletal deterioration in hPSC-CMs leading to cardiomyopathy, while on the other hand upregulates apoptosis and programmed cell death signaling as a result of DNA and mitochondrial damage.

The hPSC-CMs are specialized muscle cells which possess highly organized structures of myofilaments made up of repeating micrometer-sized units, termed sarcomeres; which are involved in mechanical cardiac contraction. Sarcomeric proteins of thin filament, thick filament and Z-disc are important in formation of basic contractile units and any alterations in their functions can result in cardiomyopathy or heart failure (Hwang and Sykes 2015). Our qRT-PCR data showed significant downregulation in mRNA levels of sarcomere genes including TCAP, MYL2, MYL3, MYH7, ACTA1, and LDB3 in hPSC-CMs treated with ETP. Immunostaining for sarcomeric proteins and transmission electron microscopy further confirmed the deterioration of myofilaments in hPSC-CMs treated with ETP compared to that of untreated CMs. High dose of ETP in particularly showed severely disorganized myofilaments.

Although regulation of $\mathrm{Ca}^{2+}$ ion homeostasis is one of the essential functional elements during cardiac contraction and is tightly regulated by $\mathrm{Ca}^{2+}$ ion channels and $\mathrm{Ca}^{2+}$ binding proteins, cytosolic $\mathrm{Ca}^{2+}$ overload leads to ischemic myocardial injury and death (Marsh and Smith 1991). The ryanodine receptors (RyRs) are intracellular calcium-release channels, expressed in cardiomyocytes and downregulation of RyRs has been reported to contribute to impaired contractility (Go et al. 1995). Our data showed clear downregulation in mRNA levels of important $\mathrm{Ca}^{2+}$ regulating genes including RyR2, CASQ2, ATP1A2 and DMD. The hiPSC-CMs treated with ETP at $10 \mu \mathrm{M}$ and $15 \mu \mathrm{M}$ showed higher $\mathrm{Ca}^{2+}$ transient amplitude, a slowing in reaching the $\left[\mathrm{Ca}^{2+}\right]$ peak as well a prolonged presence of $\mathrm{Ca}^{2+}$ in the sarcoplasm. In contrast, $30 \mu \mathrm{M}$ ETP caused an acceleration of the ascending phase of the transient. These findings further confirm that ETP has arrhythmogenic and cardiotoxic potential.

Like other DNA damaging topoisomerase inhibitors, ETP induces apoptosis in a variety of cell types and p53 has been shown to play important role in ETP-induced apoptosis (Grandela et al. 2008). Induction of apoptosis in hPSC-CMs is known to be involved in establishment of cardiotoxicity (Arola et al. 2000; Kang 2001), whereas inhibitors of apoptosis like Pifithrin- $\alpha$ have been shown to protect cardiomyocytes from DOX-induced apoptosis and acute cardiotoxicity (Liu et al. 2004) Our data show that ETP induces upregulation of genes involved in DNA damage response and apoptosis like BAX, FAS, GADD45A, and RGCC. It also leads to overall increase in ROS production in hPSC-CMs suggesting activation of apoptosis signaling. However, when hPSCCMs were treated simultaneously with ETP and Pifithrin- $\alpha$, we observed no significant improvements in hPSC-CMs viability indicating apoptosis might not be the only pathway involved in ETP-induced cardiotoxicity.

Mitochondria occupy $30 \%$ of cell volume in adult cardiomyocytes and plays essential role in cardiac muscle contraction and balance of $\mathrm{Ca}^{2+}$ and $\mathrm{K}^{+}$concentrations. Mitochondrial biogenesis involving balanced mitochondrial fission and fusion plays an important part in cardiomyocytes contractility, whereas increased mitochondrial fission leads to mitophagy as a result of increase in cytochrome c leakage (Givvimani et al. 2015; Pennanen et al. 2014). Damage to mitochondria has been shown to result in disruption of $\mathrm{Ca}^{2+}$ homeostasis and oxidative phosphorylation, increased production of reactive oxygen species (ROS), reduction in ATP production and activation of apoptosis signaling (Figueira et al. 2013; Piquereau et al. 2013; Shen and Jennings 1972). In fibroblasts, ETP induces apoptosis and cell death via mitochondrial-dependent activation of p53 (Jamil 
et al. 2015; Karpinich et al. 2002). In agreement with these observations, our data also showed increased mitochondrial fission and upregulation in SPATA18, NQO1, GLS2 and GPX1 after ETP treatment. Staining of hPSC-CMs with DHE further confirmed increased ROS production in ETPexposed hiPSC-CMs. Using JC-1 staining, we also show that ETP treatment causes alteration in mitochondrial membrane potential $(m \Delta \psi)$ indicating increased number of apoptotic hPSC-CMs and reduced ATP production. Collectively, these alterations in mitochondrial structural and functional properties indicate possible activation of ferroptosis pathway in ETP-treated CMs. Our RTCA data show that treatment of Liproxstatin-1 helps hPSC-CMs to recover from ETPinduced increase in beating rate and beating amplitude during drug washout. Further studies could shed more light on the association of ferroptosis and cardiotoxicity induced by anti-cancer drugs.

In summary, cardiovascular complications pose a rare but potential fatal adverse effect of ETP chemotherapy and should be carefully addressed, especially in patients with additional cardiovascular risk factors or when used in combination therapy. Our findings suggest that damages to mitochondria are a major contributing factor involved in ETPinduced cardiotoxicity. Even though, the exact mechanism by which ETP affects mitochondrial integrity is not fully understood, we hypothesize that damages to mitochondria and impaired mitochondrial bioenergetics and calcium handling are the major factors involved in ETP-induced cardiotoxicity. Our data also indicate that cardiotoxic effects of ETP could be synergistically increased in presence of other DNA damaging or cytotoxic agent and should be taken under consideration before starting the drug administration. In addition, in the present study, we have established an in vitro cardiotoxicity methodology for the monitoring of early as well as chronic cardiotoxicity events at the cellular, functional and genomic level.

Acknowledgements This work was supported by the 'Detection of endpoints and biomarkers for repeated dose toxicity using in vitro systems (DETECTIVE) project' (FP7 Health Programme, European Commission).

\section{Compliance with ethical standards}

Conflict of interest The authors declare no conflict of interest.

Open Access This article is distributed under the terms of the Creative Commons Attribution 4.0 International License (http://creativeco mmons.org/licenses/by/4.0/), which permits unrestricted use, distribution, and reproduction in any medium, provided you give appropriate credit to the original author(s) and the source, provide a link to the Creative Commons license, and indicate if changes were made.

\section{References}

Arola OJ, Saraste A, Pulkki K, Kallajoki M, Parvinen M, Voipio-Pulkki L-M (2000) Acute doxorubicin cardiotoxicity involves cardiomyocyte apoptosis. Cancer Res 60:1789-1792

Benov L, Sztejnberg L, Fridovich I (1998) Critical evaluation of the use of hydroethidine as a measure of superoxide anion radical. Free Radic Biol Med 25:826-831

Bernard P, Cellarier G, Cremades S, Gisserot O, De Jaureguiberry JP (2006) Early myocardial infarction associated with chemotherapy for testicular cancer. Rev Med Interne 27:497-498

Canobbio L, Fassio T, Gasparini G, Caruso G, Barzan L, Comoretto R, Brema F, Villani F (1986) Cardiac arrhythmia: possible complication from treatment with cisplatin. Tumori 72:201-204

Chan FK, Moriwaki K, De Rosa MJ (2013) Detection of necrosis by release of lactate dehydrogenase activity. Methods Mol Biol 979:65-70

Chaudhari U, Nemade H, Gaspar JA, Hescheler J, Hengstler JG, Sachinidis A (2016a) MicroRNAs as early toxicity signatures of doxorubicin in human-induced pluripotent stem cell-derived cardiomyocytes. Arch Toxicol 90:3087-3098

Chaudhari U, Nemade H, Wagh V, Gaspar JA, Ellis JK, Srinivasan SP, Spitkovski D, Nguemo F, Louisse J, Bremer S, Hescheler J, Keun HC, Hengstler JG, Sachinidis A (2016b) Identification of genomic biomarkers for anthracycline-induced cardiotoxicity in human iPSC-derived cardiomyocytes: an in vitro repeated exposure toxicity approach for safety assessment. Arch Toxicol 90:2763-2777

Chaudhari U, Ellis JK, Wagh V, Nemade H, Hescheler J, Keun HC, Sachinidis A (2017) Metabolite signatures of doxorubicin induced toxicity in human induced pluripotent stem cell-derived cardiomyocytes. Amino Acids 49:1955-1963

Chaudhari U, Nemade H, Sureshkumar P, Vinken M, Ates G, Rogiers V, Hescheler J, Hengstler JG, Sachinidis A (2018) Functional cardiotoxicity assessment of cosmetic compounds using humaninduced pluripotent stem cell-derived cardiomyocytes. Arch Toxicol 92:371-381

Choi JC, Wu W, Muchir A, Iwata S, Homma S, Worman HJ (2012) Dual specificity phosphatase 4 mediates cardiomyopathy caused by lamin A/C (LMNA) gene mutation. J Biol Chem 287:40513-40524

Cohen MH, Broder LE, Fossieck BE, Ihde DC, Minna JD (1977) Phase II clinical trial of weekly administration of VP-16-213 in small cell bronchogenic carcinoma. Cancer Treat Rep 61:489-490

Comis RL (1986) Clinical trials of cyclophosphamide, Etoposide, and vincristine in the treatment of small-cell lung cancer. Semin Oncol 13:40-44

D’Arpa P, Liu LF (1989) Topoisomerase-targeting antitumor drugs. Biochim Biophys Acta 989:163-177

Dennis G Jr, Sherman BT, Hosack DA, Yang J, Gao W, Lane HC, Lempicki RA (2003) DAVID: database for annotation, visualization, and integrated discovery. Genome Biol 4:P3

Dixon SJ, Lemberg KM, Lamprecht MR, Skouta R, Zaitsev EM, Gleason CE, Patel DN, Bauer AJ, Cantley AM, Yang WS, Morrison B, Stockwell BR (2012) Ferroptosis: an iron-dependent form of non-apoptotic cell death. Cell 149:1060-1072

Dorigo A, Mansberg R, Kwan YL (1993) Lomustine, Etoposide, methotrexate and prednisone (LEMP) therapy for relapsed and refractory non-Hodgkin's lymphoma. Eur J Haematol 50:37-40

Dweep H, Sticht C, Pandey P, Gretz N (2011) miRWalk-database: prediction of possible miRNA binding sites by "walking" the genes of three genomes. J Biomed Inform 44:839-847

Erickson SL, O’Shea KS, Ghaboosi N, Loverro L, Frantz G, Bauer M, Lu LH, Moore MW (1997) ErbB3 is required for normal cerebellar and cardiac development: a comparison with ErbB2-and heregulin-deficient mice. Development 124:4999-5011 
Escoto H, Ringewald J, Kalpatthi R (2010) Etoposide-related cardiotoxicity in a child with haemophagocytic lymphohistiocytosis. Cardiol Young 20:105-107

Ferri N, Siegl P, Corsini A, Herrmann J, Lerman A, Benghozi R (2013) Drug attrition during pre-clinical and clinical development: understanding and managing drug-induced cardiotoxicity. Pharmacol Ther 138:470-484

Figueira TR, Barros MH, Camargo AA, Castilho RF, Ferreira JC, Kowaltowski AJ, Sluse FE, Souza-Pinto NC, Vercesi AE (2013) Mitochondria as a source of reactive oxygen and nitrogen species: from molecular mechanisms to human health. Antioxid Redox Signal 18:2029-2074

Gao M, Monian P, Quadri N, Ramasamy R, Jiang X (2015) Glutaminolysis and transferrin regulate ferroptosis. Mol Cell 59:298-308

Givvimani S, Pushpakumar SB, Metreveli N, Veeranki S, Kundu S, Tyagi SC (2015) Role of mitochondrial fission and fusion in cardiomyocyte contractility. Int J Cardiol 187:325-333

Go LO, Moschella MC, Watras J, Handa KK, Fyfe BS, Marks AR (1995) Differential regulation of two types of intracellular calcium release channels during end-stage heart failure. J Clin Invest 95:888-894

Goulter AB, Goddard MJ, Allen JC, Clark KL (2004) ACE2 gene expression is up-regulated in the human failing heart. BMC Med 2:19

Grandela C, Pera MF, Wolvetang EJ (2008) p53 is required for etoposide-induced apoptosis of human embryonic stem cells. Stem Cell Res 1:116-128

Hansen M, Hirsch F, Dombernowsky P, Hansen HH (1977) Treatment of small cell anaplastic carcinoma of the lung with the oral solution of VP-16-213 (NSC 141540, 4'-demethylepipodophyllotoxin 9-(4,6-O-ethylidene-beta-D-glucopyranoside). Cancer 40:633-637

Hong WK, Nicaise C, Lawson R, Maroun JA, Comis R, Speer J, Luedke D, Hurtubise M, Lanzotti V, Goodlow J et al (1989) Etoposide combined with cyclophosphamide plus vincristine compared with doxorubicin plus cyclophosphamide plus vincristine and with high-dose cyclophosphamide plus vincristine in the treatment of small-cell carcinoma of the lung: a randomized trial of the Bristol Lung Cancer Study Group. J Clin Oncol 7:450-456

Hwang PM, Sykes BD (2015) Targeting the sarcomere to correct muscle function. Nat Rev Drug Discov 14:313-328

Jamil S, Lam I, Majd M, Tsai S-H, Duronio V (2015) Etoposide induces cell death via mitochondrial-dependent actions of p53. Cancer Cell Int 15:79

Jiang H, Pei L, Liu N, Li J, Li Z, Zhang S (2016) Etoposide-loaded nanostructured lipid carriers for gastric cancer therapy. Drug Deliv 23:1379-1382

Jougasaki M, Leskinen H, Larsen AM, Cataliotti A, Chen HH, Burnett $\mathrm{JC} \mathrm{Jr}$ (2003) Leukemia inhibitory factor is augmented in the heart in experimental heart failure. Eur J Heart Fail 5:137-145

Kang YJ (2001) Molecular and cellular mechanisms of cardiotoxicity. Environ Health Perspect 109(Suppl 1):27-34

Kang K, Oh SH, Yun JH, Jho EH, Kang J-H, Batsuren D, Tunsag J, Park KH, Kim M, Nho CW (2011) A Novel topoisomerase inhibitor, daurinol, suppresses growth of HCT116 Cells with low hematological toxicity compared to etoposide. Neoplasia (New York, NY) 13:1043-1057

Karpinich NO, Tafani M, Rothman RJ, Russo MA, Farber JL (2002) The course of etoposide-induced apoptosis from damage to DNA and p53 activation to mitochondrial release of cytochrome c. J Biol Chem 277:16547-16552

Kempf T, Wollert KC (2009) Growth differentiation factor-15: a new biomarker in cardiovascular disease. Herz 34:594-599

Kempf T, von Haehling S, Peter T, Allhoff T, Cicoira M, Doehner W, Ponikowski P, Filippatos GS, Rozentryt P, Drexler H, Anker SD, Wollert KC (2007) Prognostic utility of growth differentiation factor-15 in patients with chronic heart failure. J Am Coll Cardiol 50:1054-1060

Kitamura N, Nakamura Y, Miyamoto Y, Miyamoto T, Kabu K, Yoshida M, Futamura M, Ichinose S, Arakawa H (2011) Mieap, a p53-inducible protein, controls mitochondrial quality by repairing or eliminating unhealthy mitochondria. PLoS One 6:e16060

Klumpp TR, Mangan KF, Glenn LD, Malaspina DR, Cropper T, Mullaney M, Macdonald JS (1993) Phase I/II study of highdose cyclophosphamide, etoposide and cisplatin followed by autologous bone marrow or peripheral blood stem cell transplantation in patients with poor prognosis Hodgkin's disease or non-Hodgkin's lymphoma. Bone Marrow Transpl 12:337-345

Kridis WB, Khanfir A, Triki F, Frikha M (2013) An exceptional case of atrial fibrillation arrhythmia induced by etoposide. Curr Drug Saf 8:287-289

Kucharz J, Michalowska-Kaczmarczyk A, Zygulska AL, Wojtak J, Pawlik W, Herman RM, Krzemieniecki K (2016) Bradycardia as a rare symptom of cisplatin cardiotoxicity: a case report. Oncol Lett 11:2297-2299

Liu X, Chua CC, Gao J, Chen Z, Landy CL, Hamdy R, Chua BH (2004) Pifithrin-alpha protects against doxorubicin-induced apoptosis and acute cardiotoxicity in mice. Am J Physiol Heart Circ Physiol 286:H933-939

Marsh JD, Smith TS (1991) Calcium overload and ischemic myocardial injury. Circulation 83:709-711

Mathur A, Hong Y, Kemp BK, Barrientos AA, Erusalimsky JD (2000) Evaluation of fluorescent dyes for the detection of mitochondrial membrane potential changes in cultured cardiomyocytes. Cardiovasc Res 46:126-138

Nijmeh J, Moldobaeva A, Wagner EM (2010) Role of ROS in ischemia-induced lung angiogenesis. Am J Physiol Lung Cell Mol Physiol 299:L535-L541

Nuydens R, Novalbos J, Dispersyn G, Weber C, Borgers M, Geerts $H$ (1999) A rapid method for the evaluation of compounds with mitochondria-protective properties. J Neurosci Methods 92:153-159

Onishi Y, Azuma Y, Sato Y, Mizuno Y, Tadakuma T, Kizaki H (1993) Topoisomerase inhibitors induce apoptosis in thymocytes. Biochim Biophys Acta 1175:147-154

Pennanen C, Parra V, López-Crisosto C, Morales PE, del Campo A, Gutierrez T, Rivera-Mejías P, Kuzmicic J, Chiong M, Zorzano A, Rothermel BA, Lavandero S (2014) Mitochondrial fission is required for cardiomyocyte hypertrophy mediated by a $\mathrm{Ca}\left({ }^{2+}\right)-$ calcineurin signaling pathway. J Cell Sci 127:2659-2671

Petrella V, Alciato P, Cantone PA, Fico D, Gagliardini R (1989) High-frequency atrial arrhythmia induced by a cisplatin-etoposide combination. Minerva Med 80:305-307

Piquereau J, Caffin F, Novotova M, Lemaire C, Veksler V, Garnier A, Ventura-Clapier R, Joubert F (2013) Mitochondrial dynamics in the adult cardiomyocytes: which roles for a highly specialized cell? Front Physiol 4:102

Rodriguez J, Collazos J, Gallardo M, Hernando G (1995) Angina pectoris following cisplatin, etoposide, and bleomycin in a patient with advanced testicular cancer. Ann Pharmacother 29:138-139

Schmoll HJ, Niederle N, Achterrath W (1981) Etoposide VP 16-213)--a podophyllotoxinderivative with high antitumor activity (author's transl). Klin Wochenschr 59:1177-1188

Schwarzer S, Eber B, Greinix H, Lind P (1991) Non-Q-wave myocardial infarction associated with bleomycin and etoposide chemotherapy. Eur Heart J 12:748-750

Shen AC, Jennings RB (1972) Myocardial calcium and magnesium in acute ischemic injury. Am J Pathol 67:417-440

Shoji S, Miyakita H, Shima M, Usui Y, Nagata Y, Uchida T, Terachi T (2006) Acute myocardial infarction during combined 
chemotherapy with bleomycin, etoposide and cisplatin for testicular cancer. Hinyokika Kiyo 52:723-726

Soubeyrand S, Pope L, Hache RJ (2010) Topoisomerase IIalphadependent induction of a persistent DNA damage response in response to transient etoposide exposure. Mol Oncol 4:38-51

Tran MK, Kurakula K, Koenis DS, de Vries CJM (2016) Proteinprotein interactions of the LIM-only protein FHL2 and functional implication of the interactions relevant in cardiovascular disease. Biochim Biophys Acta Mol Cell Res 1863:219-228

Walker PR, Smith C, Youdale T, Leblanc J, Whitfield JF, Sikorska M (1991) Topoisomerase II-reactive chemotherapeutic drugs induce apoptosis in thymocytes. Cancer Res 51:1078-1085

Willmore E, Frank AJ, Padget K, Tilby MJ, Austin CA (1998) Etoposide targets topoisomerase IIalpha and IIbeta in leukemic cells: isoform-specific cleavable complexes visualized and quantified in situ by a novel immunofluorescence technique. Mol Pharmacol $54: 78-85$
Xie Y, Hou W, Song X, Yu Y, Huang J, Sun X, Kang R, Tang D (2016) Ferroptosis: process and function. Cell Death Differ 23:369-379

Yan X, Morgan JP (2011) Neuregulin1 as novel therapy for heart failure. Curr Pharm Des 17:1808-1817

Yano S, Shimada K (1996) Vasospastic angina after chemotherapy by with carboplatin and etoposide in a patient with lung cancer. Jpn Circ J 60:185-188

Yeh ET, Tong AT, Lenihan DJ, Yusuf SW, Swafford J, Champion C, Durand JB, Gibbs H, Zafarmand AA, Ewer MS (2004) Cardiovascular complications of cancer therapy: diagnosis, pathogenesis, and management. Circulation 109:3122-3131

Zhao L, Zhang B (2017) Doxorubicin induces cardiotoxicity through upregulation of death receptors mediated apoptosis in cardiomyocytes. Sci Rep 7:44735 